\title{
General relativistic radiative transfer code in rotating black hole space-time: ARTIST
}

\author{
Rohta Takahashi ${ }^{1 \star} \dagger$ and Masayuki Umemura ${ }^{2}$ \\ ${ }^{1}$ National Institute of Technology, Tomakomai College, 443 Nishikioka, Tomakomai, Hokkaido 059-1275, Japan \\ ${ }^{2}$ Center for Computational Sciences, University of Tsukuba, 1-1-1 Tennodai, Tsukuba, Ibaraki 305-8577, Japan
}

Accepted 2016 September 29. Received 2016 September 26; in original form 2016 June 1

\begin{abstract}
We present a general relativistic radiative transfer code, ARTIST (Authentic Radiative Transfer In Space-Time), that is a perfectly causal scheme to pursue the propagation of radiation with absorption and scattering around a Kerr black hole. The code explicitly solves the invariant radiation intensity along null geodesics in the Kerr-Schild coordinates, and therefore properly includes light bending, Doppler boosting, frame dragging, and gravitational redshifts. The notable aspect of ARTIST is that it conserves the radiative energy with high accuracy, and is not subject to the numerical diffusion, since the transfer is solved on long characteristics along null geodesics. We first solve the wavefront propagation around a Kerr black hole that was originally explored by Hanni. This demonstrates repeated wavefront collisions, light bending, and causal propagation of radiation with the speed of light. We show that the decay rate of the total energy of wavefronts near a black hole is determined solely by the black hole spin in late phases, in agreement with analytic expectations. As a result, the ARTIST turns out to correctly solve the general relativistic radiation fields until late phases as $t \sim 90 \mathrm{M}$. We also explore the effects of absorption and scattering, and apply this code for a photon wall problem and an orbiting hotspot problem. All the simulations in this study are performed in the equatorial plane around a Kerr black hole. The ARTIST is the first step to realize the general relativistic radiation hydrodynamics.
\end{abstract}

Key words: black hole physics-radiative transfer-relativistic processes-methods: numerical.

\section{INTRODUCTION}

The relativistic effects of radiation play a significant role in accretion/outflow around a black hole. The structure of black hole accretion discs is dependent on optical depth and mass accretion rate that include a radiatively inefficient accretion flow (RIAF) with the mass accretion rate much lower than the Eddington rate, a geometrically-thin standard disc (Shakura \& Sunyaev 1973) with a nearly Eddington accretion rate, and a geometrically-, opticallythick disc (Abramowicz et al. 1988; Abramowicz \& Fragile 2013) with a super-Eddington accretion rate. In a standard disc, while angular momentum is transported via magnetorotational instability (Balbus \& Hawley 1991, 1998) and outflows are driven by magnetohydrodynamical mechanism (Blandford \& Begelman 1999; Ohsuga $\&$ Mineshige 2014), the radiative transfer (RT) regulates the thermal properties via cooling and heating and therefore the dynamical

\footnotetext{
*E-mail: takahashi@ tomakomai-ct.ac.jp

$\dagger$ Also, a visiting scientist of High Energy Astrophysics Laboratory, RIKEN,

a visiting associate professor of Nagaoka University of Technology.
}

structure. In a disc with super-Eddington accretion rate, since photons are tightly coupled with fluid (so-called photon-trapping), photons are swallowed by a black hole without diffusing out from the vicinity of a black hole (Begelman 1978; Houck \& Chevalier 1991; Ohsuga \& Mineshige 2007, 2011). Hence, the super-Eddington mass accretion is thought to be retained via such photon-trapping (Ohsuga \& Mineshige 2007; McKinney et al. 2014). In the photontrapping, the RT of scattered photons around a black hole plays a key role. However, the RT in the general relativistic (GR) regime has not been hitherto solved precisely. Besides, it is revealed by recent GR magnetohydrodynamic simulations that the effects of radiative cooling are essential even for a RIAF in Sgr A* or M87 (Mościbrodzka et al. 2011; Dibi et al. 2012; Mościbrodzka et al. 2014; Mościbrodzka, Falcke \& Shiokawa 2016). Hence, in a wide range of the black hole accretion, solving the RT equation in black hole space-time is of great significance.

In the Minkowski space-time, time-independent RT solvers (Stone, Mihalas \& Norman 1992; Hayes \& Norman 2003; Hubeny \& Burrows 2007; Davis, Stone \& Jiang 2012) or Monte Carlo methods (Yusef-Zadeh, Morris \& White 1984; Densmore et al. 2007; Whitney 2011; Abdikamalov et al. 2012; Steinacker, Baes 
\& Gordon 2013) have been developed so far. Also, neutrino RT simulations have been performed in core-collapsed supernovae (e.g. Mezzacappa \& Bruenn 1993; Mezzacappa et al. 2001; Liebendörfer et al. 2005), and multidimensional neutrino RT equations are solved based on Eulerian formulation by using the discrete-ordinate $S_{n}$ method (Pomraning 1973; Morel et al. 2003; Sumiyoshi et al. 2005; Sumiyoshi, Yamada \& Suzuki 2007; Ott et al. 2008; Sumiyoshi \& Yamada 2012; Nagakura, Sumiyoshi \& Yamada 2014; Sumiyoshi et al. 2015) and spectral methods (Peres et al. 2014). As an alternative to these listed RT methods, the collisionless Boltzmann (Vlasov) equation has also been solved in six-dimensional Eulerian grids of phase space (Yoshikawa, Yoshida, \& Umemura 2013).

In the cosmological simulations, the RT equation has been solved in the three-dimensional space with various numerical methods (e.g. Iliev et al. 2006, 2009; Okamoto, Yoshikawa \& Umemura 2012). The most accurate method is a long characteristic (LC) method (Abel, Norman \& Madau 1999; Sokasian, Abel \& Hernquist 2001; Susa 2006). In most of LC methods, all meshes that have photon sources are directly connected to each other by ray-tracing. Therefore, this method is highly time-consuming computationally. On the other hand, a short characteristic (SC) method reduces the computational cost by solving RT for adjacent meshes (Kunasz \& Auer 1988; Stone et al. 1992; Mellema et al. 1998; Nakamoto, Umemura $\&$ Susa 2001). However, the SC method suffers from non-negligible numerical diffusion, so it is hard to track a collimated radiation field and delineate a sharp shadow. A novel method retaining the accuracy of an LC method with computational cost as low as that of an SC method was proposed by Razoumov \& Cardall (2005) and Nakamoto, Susa \& Umemura in Iliev et al. (2006). The method by Nakamoto, Susa \& Umemura is called an authentic RT (ART) method. In the ART method, it is demonstrated that the collimated radiation field is accurately solved without numerical diffusion with computational cost similar to the SC method. Other methods include tree- or oct-tree-based accelerated RT method (Hasegawa \& Umemura 2010; Okamoto et al. 2012) as well as Monte Carlo methods (Ciardi et al. 2001).

In various astrophysical issues, the RT should be coupled with hydrodynamics. In such radiation hydrodynamics (RHD) calculations, one often invokes a closure relation to solve the full set of RHD equations. In the past simulations, approximate closure relations such as the flux-limited diffusion (Levermore \& Pomraning 1981; Turner \& Stone 2001; Castor 2004), M1 closure (Levermore 1984; Dubroca \& Feugeas 1999; González, Audit \& Huynh 2007; Sądowski et al. 2013; Skinner \& Ostriker 2013; Takahashi \& Ohsuga 2013; McKinney et al. 2014; Takahashi \& Ohsuga 2015), Monte Carlo method (Ryan, Dolence \& Gammie 2015), photon-conserving scheme (Sajowski \& Narayan 2015, 2016) or the truncated moment formalism (Shibata \& Sekiguchi 2012) were employed. Recently, the relativistic time-dependent RT equation is directly solved (Jiang, Stone \& Davis 2012, 2013, 2014; Zhu et al. 2015; Jiang, Davis \& Stone 2016; Narayan et al. 2016; Ohsuga \& Takahashi 2016), which is an important step towards full GR approach.

Although a multitude of RT codes in the Minkowski space-time are available, no time-dependent GR RT scheme has been developed as of yet. In this paper, we present an RT code in curved space-time that is a GR extension of the ART method. First, we construct a geodesic generator based on mathematic formulas of geodesics, MASTER (MAthematic Space-Time Emulator with Raytracing), that provides geodesic coordinates by the ray-tracing along the LCs of null geodesics in curved space-time. Then, we develop a GR version of the ART code, ARTIST (Authentic Radiative Transfer In Space-Time), that solves the time-dependent RT along the null geodesics generated with MASTER. This code is not subject to numerical diffusion and therefore properly incorporates GR effects like light bending, Doppler boosting, frame-dragging and gravitational redshifts. In the original ART method, the steady-state radiation transfer is solved in the non-relativistic regime by dismissing the time-derivative term. However, the ARTIST solves the time-dependent RT equation, retaining the causality of photon propagation. Therefore, we modify the original ART method to trace the light propagating along the geodesics with the speed of light. In this study, the propagation of wavefronts in curved space-time is tested, and it is shown that the ARTIST correctly solves the GR radiation fields conserving the energy even in the late phase as $t \sim 90 M$. The test problems proposed in this study are useful as benchmarks of the GR RT simulations in the future.

The structure of the paper is as follows. We describe our GR RT code, ARTIST, in Section 2. In Section 3, we present the results of the RT simulations with ARTIST. Section 4 is devoted to conclusions. We present the numerical method and the code verifications of MASTER in Appendix A. Throughout the paper, we use the geometric units $c$ $=G=1$ and the signature of space-time $(-,+,+,+)$.

\section{GR RT CODE: ARTIST}

In this section, we describe general features and numerical methods of our GR RT code: ARTIST. The code consists of three main components.

(i) The first component is a null geodesic generator MASTER, that provides the geodesic coordinates in curved space-time by solving the equation of null geodesics as

$\frac{\mathrm{d} x^{\mu}}{\mathrm{d} \lambda}=k^{\mu}, \quad \frac{\mathrm{d} k_{\mu}}{\mathrm{d} \lambda}=-\frac{1}{2} \frac{\partial g^{\alpha \beta}}{\partial x^{\mu}} k_{\alpha} k_{\beta}$,

where $\lambda$ is the affine parameter, and $x^{\mu}$ and $k^{\mu}$ are, respectively, the coordinates and the four momentum of photons. The null geodesics obtained with MASTER are the LCs in the curved space-time. The details and the verifications of MASTER are summarized in Appendix A. The null geodesics set up by MASTER are used for RT calculations by the ARTIST. See Section 2.1 for details.

(ii) The second component is a GR RT solver that solves the transfer equation of invariant specific intensity $\mathcal{I}$ as

$\frac{\mathrm{d} \mathcal{I}}{\mathrm{d} \lambda}=\mathcal{E}-\mathcal{A I}$,

(e.g. Lindquist 1966; Sachs \& Ehlers 1968; Ehlers 1971; Israel 1972; Mihalas \& Mihalas 1984; Cardall, Lentz \& Mezzacappa 2005; Vincent et al. 2011; Younsi, Wu \& Fuerst 2012; Cardall, Endeve \& Mezzacappa 2013) where $\mathcal{E}$ is the invariant emissivity consisting of pure emission and incoming scattered photons and $\mathcal{A}$ is the invariant absorption coefficients consisting of pure absorption and contributions by outgoing scattering photons. The RT equation is solved along the null geodesics generated by MASTER. The invariant physical quantities (the invariant specific intensity $\mathcal{I}$, the invariant emissivity $\mathcal{E}$ and the invariant absorption coefficient $\mathcal{A}$ ) satisfy the following relations

$\mathcal{I}=\frac{I_{v}}{v^{3}}, \quad \mathcal{E}=\frac{j_{v}}{v^{2}}, \quad \mathcal{A}=v \alpha_{v}$,

where $v, I_{v}, j_{v}$ and $\alpha_{v}$ are photon frequency (energy), specific intensity, emissivity and extinction coefficient (including scattering) measured in the local Minkowski frame. For the invariant intensity solver, see Section 2.3 for details. 
(iii) The third component is the radiation tensor calculator that figures the radiation stress-energy tensor $R^{\mu \nu}$ by the integration of the invariant intensity $\mathcal{I}$ in the momentum space of radiation. In this component, the radiation tensor in the coordinate frame $R^{\mu v}$ is calculated as follows. First, the radiation tensor $R^{(\mu)(v)}$ in the locally non-rotating reference frame (LNRF; e.g. Bardeen, Press \& Teukolsky 1972; Wald 1984; Frolov \& Novikov 1998) is evaluated:

$R^{(\alpha)(\beta)}=\int \mathcal{I} k^{(\alpha)} k^{(\beta)} k^{(0)} \mathrm{d} k^{(0)} \mathrm{d} \Omega$,

where the parenthesis denotes the quantities in the LNRF and $\mathrm{d} \Omega=$ $\sin \bar{\theta} \mathrm{d} \bar{\theta} \mathrm{d} \bar{\phi}$ with the angular coordinates $(\bar{\theta}, \bar{\phi})$. In this integration, the four vector in the LNRF, $k^{(\alpha)}$, is calculated from the four vector in the coordinate frame, $k^{\mu}$, that is provided by the geodesic generator MASTER. Then, we transform $R^{(\alpha)(\beta)}$ to the radiation stress tensor $R^{\mu v}$ in the coordinate frame using the LNRF tetrad vectors $e_{(\alpha)}^{\mu}$ as

$R^{\mu \nu}=e_{(\alpha)}^{\mu} e_{(\beta)}^{\nu} R^{(\alpha)(\beta)}$.

In this study, we use the tetrad vectors in the LNRF in the Kerr space-time written in the Kerr-Schild (KS) coordinates. These vectors are given in the past studies by (King, Lasota \& Kundt 1975; Gourgoulhon \& Jaramillo 2006; Takahashi 2007, 2008; Zhang et al. 2012). We find that the accurate calculations of the momentum integration in equation (4) are required for the energy-conserved RT simulations. See Section 2.2 for details.

The numerical procedures of these components are described in more details in the following.

\subsection{Coordinates in the phase space of radiation}

We generate the geodesic coordinates along the null geodesics in curved space-time with MASTER. In the black hole space-time, it seems practically hard to completely cover the phase space, since there are extremely relativistic orbits skimming the photon sphere and circling many times around the black hole. However, extremely relativistic orbits are quite rare, because they are engendered in very narrow solid angles. Hence, we generate all patterns of null geodesics except the extremely relativistic orbits, and set the geodesic coordinates in curved space-time.

The null geodesics around a rotating black hole are classified into four types shown in Fig. 1 (e.g. Čadež \& Kostić 2005); (a) scattering orbits with both end points at infinity, (b) incoming orbits with a start point at infinity and an end point inside the horizon, (c) outgoing orbits with a start point in the vicinity of the horizon and an end point at infinity, (d) closed orbits with a start point in the vicinity of the horizon and an end point inside the horizon. These orbits are calculated in the KS coordinates. In Fig. 1, the black hole rotation is counter-clockwise as shown by a curved arrow, and the directions of the propagation of photons along geodesics are denoted by arrows. ${ }^{1}$ Because the closed orbits are located inside the

\footnotetext{
${ }^{1}$ It is noted that for the geodesics in the Boyer-Lindquist (BL) coordinates, the shapes of incoming orbits and outgoing orbits are the same except the directions of the propagation. In this case, the null geodesics in Schwarzschild space-time are classified into (i) scattering orbit, (ii) plunging orbit and (iii) closed orbit (near orbit) (e.g. Čadež \& Kostić 2005). In the KS case, outside the event horizon, the incoming orbits and the outgoing orbits are related by simple analytical relations. Therefore, the incoming (outgoing) orbit can be obtained from the outgoing (incoming) by the simple analytic transformations.
}

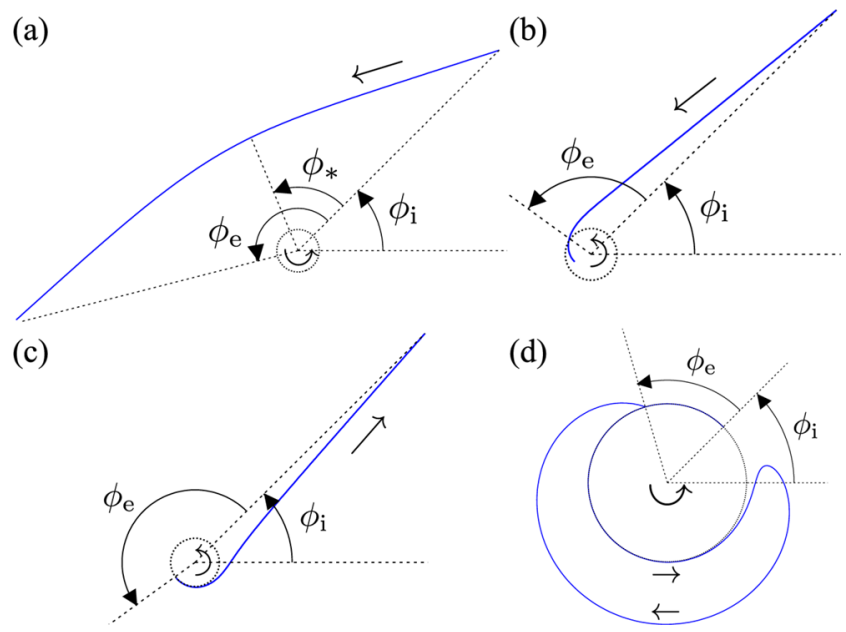

Figure 1. Schematic patterns of null geodesics around a rotating black hole: (a) scattering orbit, (b) incoming orbit, (c) outgoing orbit, and (d) closed orbit. The black hole rotation is counter-clockwise shown by a curved arrow. The directions of the photon propagation are shown by arrows. The event horizon is shown by a dotted circle. In these plots, $\phi_{\mathrm{i}}$ and $\phi_{\mathrm{e}}$ denote $\phi$ coordinates of the end points of the null geodesics. In the panel (a), $\phi_{*}$ denotes $\phi$-coordinate of the intermediate point on the null geodesics.

photon sphere, they become important when photons are emitted or scattered in the photon sphere.

In the GR RT simulations in this study, we perform the simulations in the equatorial plane and use the equatorial geodesics. Also, simulations are done in the region whose outer boundary is at $r$ $=20 M$ and inner boundary at $r=0.7 r_{+}$, where $r_{+}$is the outer horizon of the Kerr black hole. For scattering orbits in the panel (a) of Fig. 1, we calculate the geodesics from the outer boundary by shooting many rays. In this figure, $\phi_{\mathrm{i}}$ and $\phi_{\mathrm{e}}$ denote the angles of the start and end points at the outer boundary, respectively. ${ }^{2}$ Incoming orbits, as shown in the panel (b) of Fig. 1, are calculated in the same way as the scattering orbits except that the end point is located inside the event horizon. For these orbits, the geodesic equations are solved forward in time. On the other hand, outgoing orbits in the panel (c) of Fig. 1 are calculated from the outer boundary by solving the geodesic equations backwards in time as shown by $\phi_{\mathrm{i}}$ and $\phi_{\mathrm{e}}$. For outgoing orbits, null geodesics are calculated in the region between $r=r_{+}+0.01 M$ and $r_{+}+0.1 M$. Closed orbits in the panel (d) of Fig. 1 are calculated in the same way except that the start points of geodesics are set near or inside the event horizon. In the numerical calculations of the geodesics, the initial angular coordinates $(\bar{\theta}, \bar{\phi})$ are given in the LNRF at the initial position $x^{\mu}$.

Next, we describe how the geodesic coordinates are settled. We use the geodesics within the range of $0 \leq \phi_{\mathrm{i}} \leq 2 \pi$ and $-6 \pi \leq$ $\phi_{\mathrm{e}} \leq 6 \pi$ for the four types of orbits. The angles $\phi_{i}$ and $\phi_{\mathrm{e}}$ are equally discretized. The number of the discretized angle for $\phi_{i}$ is $N_{\phi_{\mathrm{i}}}=256$. On the other hand, the number of the discretized angle for $\phi_{\mathrm{e}}$ is $N_{\phi_{\mathrm{e}}}=256 \times 6$ for scattering orbits and $128 \times 6$ for incoming, outgoing and closed orbits. Along each geodesic, the geodesic coordinates are set with equal intervals of $\Delta t=0.2 \mathrm{M}$. It is noted that this interval can be arbitrarily changed depending on spatial grids, as far as the memory of the computational facilities allows. In this study, we only perform the GR RT simulations with the fixed interval. To accelerate the geodesic calculations, we partly

\footnotetext{
2 The angle $\phi_{*}$ denotes the angular position of the intermediate point along
} the ray that is used later (in Fig. 3). 
use the graphics processing unit (GPU), NVIDIA Tesla K20, with double precision. ${ }^{3}$

\subsection{Energy-conserving scheme for integration in momentum space}

In this subsection, we describe the numerical method of the integration in the momentum space used in ARTIST.

The specific intensity is integrated in the momentum space to calculate the radiation stress tensor and to evaluate the scattering term in the RT equation. An energy-conserving scheme is required in this momentum integration to obtain the proper results in long-term simulations. We find that simple algorithms do not conserve the radiation energy and therefore the GR effects cannot be traced correctly. Here, we concentrate our attention on properly incorporating the GR effects in long-term simulations.

The integration in the momentum space is performed in the LNRF as equation (4). Then, we obtain the radiation tensor in the coordinate frame by equation (5). The radiation tensor is calculated on the spatial coordinates in the range of $r_{+} \leq r \leq 20 M$ and $0 \leq \phi \leq 2 \pi$ in the equatorial plane. We use the spherical angular coordinates to evaluate of radiation tensor.

In Fig. 2, we present the schematic illustration of (1) the ART method and (2) the present scheme, ARTIST. In both schemes, the radiation stress-energy tensor is calculated with invariant specific intensities inside a spatial grid (denoted by the triangle in Fig. 2). The radiation stress-energy tensor is evaluated with specific intensities on the nearest neighbours denoted by the grey circle. In this figure, the spatial grids are shown by dashed lines and the positions of grid centres are denoted by the index $(i, j)$. The null geodesics are shown by solid lines.

In the ART method (shown in the panel a in Fig. 2), the radiation tensor is evaluated by the interpolations of specific intensities (shown by the grey circle) on the nearest neighbour grid lines around the grid (shown by the triangle).

In the present scheme, ARTIST (shown in the panel b in Fig. 2), the radiation tensor is evaluated with the nearest neighbour specific intensity (denoted by the grey circle) in the grid zone whose boundary is shown by the black solid line. In the panel (b), the geodesics are labelled with the index $k_{*}$. The geodesic coordinates are specified by the index $i_{*}$. The radiation tensor $R^{(\alpha)(\beta)}$ in the LNRF given by equation (4) is evaluated as

$\left[R^{(\alpha)(\beta)}\right]_{i, j}=\sum_{k=1}^{N_{k}} \mathcal{I}_{i, j, k} k_{i, j, k}^{(\alpha)} k_{i, j, k}^{(\beta)} k_{i, j, k}^{(0)} \Delta k_{i, j, k}^{(0)} \Delta \Omega_{i, j, k}$,

where $k$ is the index of geodesic coordinates inside a grid and $N_{k}$ is the number of such geodesic coordinates in the grid zone. In order to preserve the causality in the RT simulations with scattering, for one geodesic line, one nearest neighbour specific intensity is used in the calculation of equation (6).

A naive evaluation is made using $\mathcal{I}_{i, j, k}, k_{i, j, k}^{(\mu)}, \Delta k_{i, j, k}^{(0)}$ and $\Delta \Omega_{i, j, k}$ at the geodesic coordinates inside a grid. However, as shown below, such an evaluation does not conserve the radiation energy. This comes from the fact that $k_{i, j, k}^{(\mu)}, \Delta k_{i, j, k}^{(0)}$ and $\Delta \Omega_{i, j, k}$ on the geodesic coordinates are slightly different from those at the grid centre. This difference adds the artificial energy to the radiation. Therefore, we should improve the scheme to conserve the radiation energy on the

\footnotetext{
${ }^{3}$ The details of the computational performances in the geodesics calculations by GPU will be reported in future studies.
}

(a)

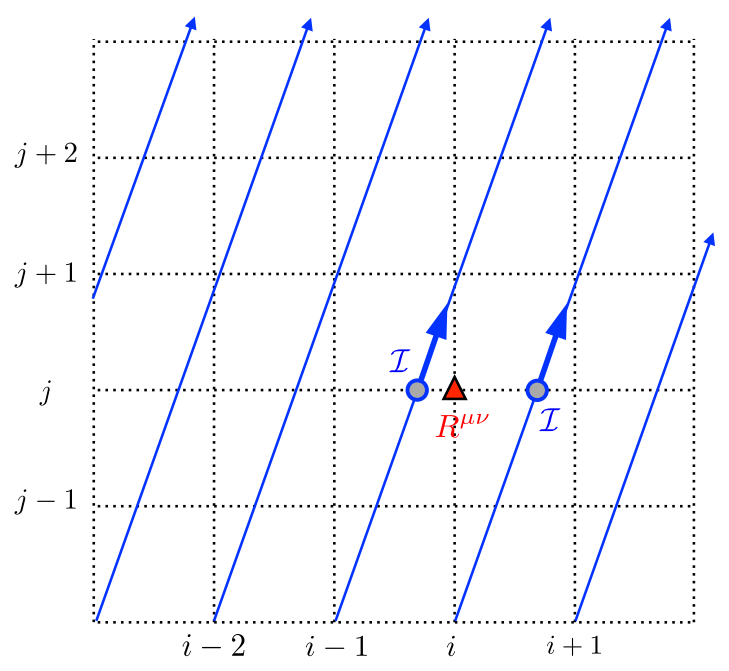

(b)

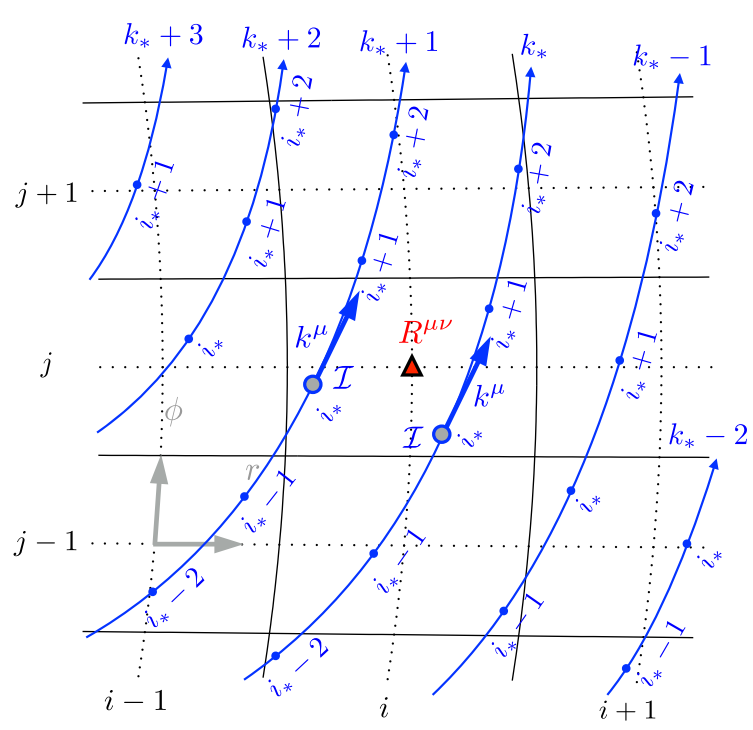

Figure 2. Schematic illustration of radiative transfer calculations in (a) the ART method and (b) the present scheme, ARTIST. (a) The spatial grids are shown by dotted lines. The radiative transfer equation is solved along parallel light rays (blue lines) and the radiation stress-energy tensor, $R^{\mu \nu}$, is evaluated by the interpolation of specific intensities on the nearest neighbours denoted by grey circles. (b) The spatial grids are shown by dotted curves. The geodesics are represented by blue curves labelled with $k_{*}$, and the geodesic coordinates are denoted by $i_{*}$. Invariant specific intensities, $\mathcal{I}$, are obtained by transfer calculations along geodesics. The radiation stressenergy tensor, $R^{\mu \nu}$, is evaluated from the specific intensities inside a grid specified by index $(i, j)$. The boundaries of the spatial grid are shown by the solid curves. For example, $R^{\mu v}$ at the point of a triangle is evaluated from specific intensities inside a spatial grid that are shown by grey circles with arrows. These arrows show the direction of the propagation of radiation. The integration over solid angles of $R^{\mu \nu}$ is performed for geodesics with different directions.

momentum integration. Later, we call the scheme with this naive evaluation 'simple method' (e.g. in Fig. 8).

In this study, we introduce a novel technique for the integration in the momentum space. The essential point is that $k_{i, j, k}^{(\mu)}, \Delta k_{i, j, k}^{(0)}$ and $\Delta \Omega_{i, j, k}$ are calculated on the null geodesic that exactly passes a grid centre. Such geodesic is calculated for every grid centre, using the geodesic generator MASTER. In Fig. 3, we illustrate the geodesics passing a grid centre. The panel (a) of Fig. 3 shows the 
(a)

(b)
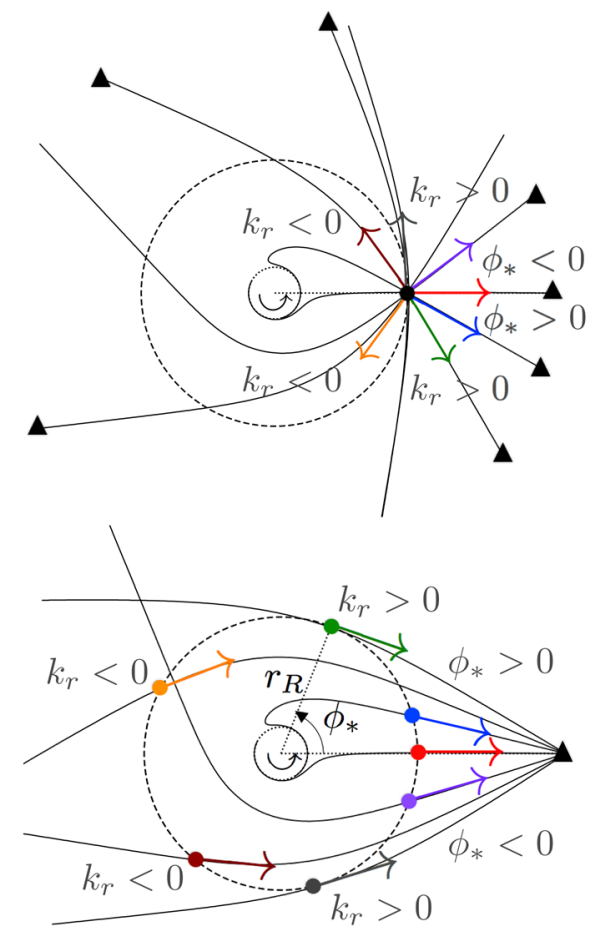

(c)

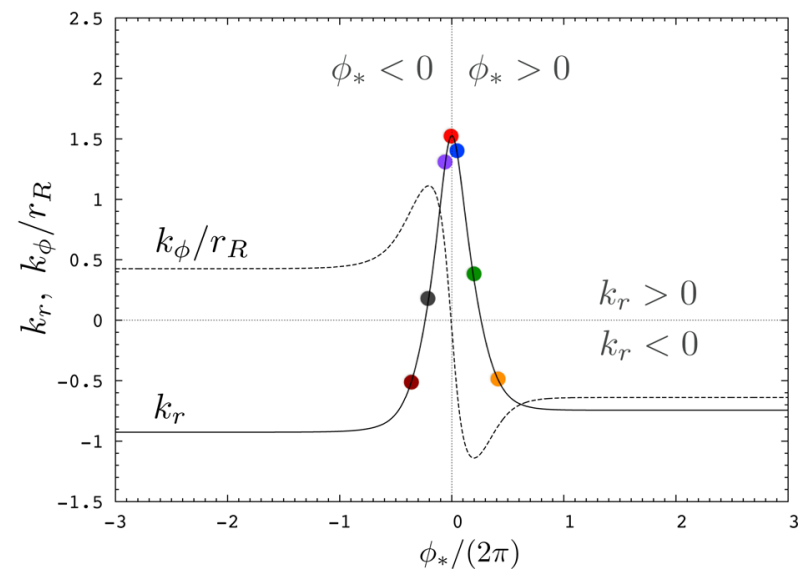

Figure 3. (a) Schematic illustration of the patterns of null geodesics passing a grid centre (filled circle). The end points of null geodesics are shown by filled triangles. (b) Rearrangement of geodesics shown in panel (a) with the same end point. (c) The photon four momenta of geodesics passing a grid centre as a function of angles $\phi_{*}$ that are shown in panel (a) in Fig. 1. Coloured dots correspond to those in panel (b).

geodesic patterns passing a grid centre denoted by a filled circle. The end points of geodesics are denoted by filled triangles. These geodesics can be rearranged with the same end point as shown in the panel (b). In the panel (c), the photon four momenta at the grid centre are presented as a function of angles $\phi_{*}$ that are shown in panel (a) in Fig. 1. In ARTIST, $k_{i, j, k}^{(\mu)}, \Delta k_{i, j, k}^{(0)}$ and $\Delta \Omega_{i, j, k}$ in equation (6) are calculated from the null geodesics passing a grid centre as shown in Fig. 3.

In Fig. 4 , we compare radial components $\left(k_{\mathrm{r}}\right)$, the photon four momenta between a simple method and an improved method used in ARTIST. The inset of Fig. 4 is the zoom-in diagram in the range of $0.4 \leq \phi_{*} / 2 \pi \leq 0.5$. The $k^{\mathrm{r}}$-components of the geodesic that exactly passes a grid centre are shown by a red solid line connected with red

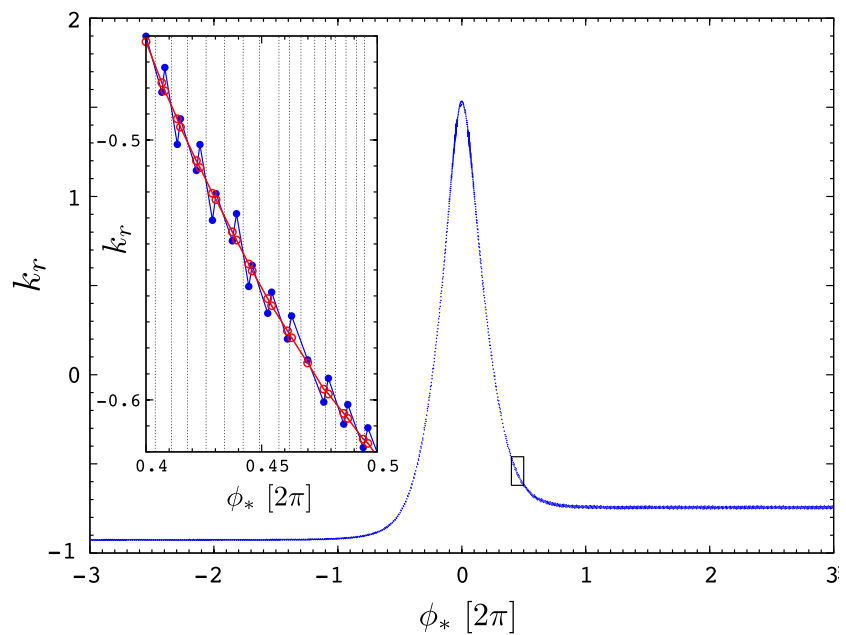

Figure 4. Examples of radial components, $k_{\mathrm{r}}$, of the photon four momentum of the nearest neighbours around a grid. The inset is the zoom-in diagram in the range of $0.4 \leq \phi_{*} / 2 \pi \leq 0.5$. The $k_{\mathrm{r}}$-components of the geodesic passing a grid centre are shown by a red solid line with red open circles, while the $k_{\mathrm{r}}$-components on the geodesic coordinates on the nearest geodesics inside a grid zone are shown by a blue line with blue filled circles.

open circles, while the $k^{r}$-components on the geodesic coordinates on the nearest geodesics inside a grid are shown by a blue line connected with blue filled circles. As shown in this figure, blue filled circles are scattered around a red line. This is simply because the null geodesics of the nearest neighbors do not pass the grid center. In the simple method, the photon four momentum on the geodesics passing the grid centre is estimated from the photon four momentum in the nearest neighbour geodesic grid with linear interpolations. ${ }^{4}$

On the other hand, in ARTIST, the four momenta $k_{i, j, k}^{(\mu)}$ in equation (6) are replaced by those of the geodesics passing a grid centre. These replacements are made for all the grids where the radiation tensor is calculated. With the replaced photon four momenta, we calculate $\Delta k_{i, j, k}^{(0)}$ and $\Delta \Omega_{i, j, k}$. As shown later (in Fig. 8), this improvement leads to dramatically high accuracy of energy conservation that allows us to simulate the wavefronts correctly even after many rotations in a BH metric. We also use this scheme for the calculation of the scattering term of the RT equation as presented below.

\subsection{Solver of the invariant specific intensity}

Here, we describe our solver of the RT equation (2). The invariant specific intensity, $\mathcal{I}$, is transported along the geodesics with the speed of light $c .^{5}$ In the present code, we set the timestep, $\Delta t$, to be constant, and therefore the geodesic coordinates are determined by the relation of $\Delta \xi^{i_{*}}=c \Delta t$, where $\Delta \xi^{i_{*}}$ is the separation between $i_{*}$ th point and $\left(i_{*}+1\right)$ th point

We first consider a case of pure emission without absorption and scattering, i.e. in equation (2), we set $\mathcal{A}=0$ and the pure emission term only in $\mathcal{E}$. The affine parameter $\lambda$ in equation (2) is converted to the normal time $t$ as $\mathrm{d} t=k^{\mathrm{t}} \mathrm{d} \lambda$, where $k^{\mathrm{t}}$ is the time component

\footnotetext{
${ }^{4}$ We have checked that even with second-order interpolations, the radiation energy in the RT simulations is not conserved.

${ }^{5}$ In this paragraph, we explicitly write the speed of light as $c$. In other parts of this paper, we use the unit of $c=1$.
} 
of the photon four momentum. Then, the RT equation is simply written as

$\mathrm{d} \mathcal{I} / \mathrm{d} t=\mathcal{E} / k^{\mathrm{t}}$

To solve this time-dependent RT equation, we make time-explicit discretization:

$\mathcal{I}_{k_{*}}^{i_{*}+1}=\mathcal{I}_{k_{*}}^{i_{*}}+\Delta t\left(\mathcal{E} / k^{\mathrm{t}}\right)_{k_{*}}^{i_{*}}$,

where $k_{*}$ specifies a geodesic and $i_{*}$ refers to the geodesic coordinate. This discretization is made along the null geodesics calculated by MASTER.

Next, we incorporate the absorption and the scattering. In general, the invariant emissivity $\mathcal{E}$ consists of the pure emission $\mathcal{E}_{\mathrm{e}}$ and the incoming scattered photons $\mathcal{E}_{\mathrm{s}}$. Similarly, the invariant absorption coefficients $\mathcal{A}$ consists of the pure absorption coefficient $\mathcal{A}_{\mathrm{a}}$ and the scattering coefficient $\mathcal{A}_{\mathrm{s}}$ for outgoing photons. Then, we have

$\mathcal{E}=\mathcal{E}_{\mathrm{e}}+\mathcal{E}_{\mathrm{s}}, \quad \mathcal{A}=\mathcal{A}_{\mathrm{a}}+\mathcal{A}_{\mathrm{s}}$.

The albedo for scattering $\varpi$ is defined as

$\varpi=\frac{\mathcal{A}_{\mathrm{s}}}{\mathcal{A}_{\mathrm{a}}+\mathcal{A}_{\mathrm{s}}}$.

With albedo $\varpi$, the pure absorption coefficient $\mathcal{A}_{\mathrm{a}}$ and the scattering coefficient $\mathcal{A}_{\mathrm{s}}$ for outgoing photons are calculated as

$\mathcal{A}_{\mathrm{s}}=\varpi \mathcal{A}, \quad \mathcal{A}_{\mathrm{a}}=(1-\varpi) \mathcal{A}$.

The invariant emissivity due to incoming photons is given by the sum of the scattered photons coming from all directions in momentum space as

$\mathcal{E}_{\mathrm{s}}\left(x^{\mu}, k^{\mu}\right)=\int \mathrm{d} P^{\prime} \mathcal{A}_{\mathrm{s}}\left(x^{\mu}, k^{\prime \mu}\right) \mathcal{I}\left(x^{\mu}, k^{\prime \mu}\right) \zeta\left(x^{\mu} ; k^{\prime \mu} \rightarrow k^{\mu}\right)$,

where the function $\zeta\left(x^{\mu} ; k^{\prime \mu} \rightarrow k^{\mu}\right)$ is called the invariant phase function (Chandrasekhar 1950; Lindquist 1966) that represents the increase of photons due to scattering into a four momentum range $\mathrm{d} P$ out of all four momentum ranges $\mathrm{d} P^{\prime}$. Therefore, the RT equation is described as (Lindquist 1966)

$$
\begin{aligned}
& \frac{\mathrm{d} \mathcal{I}\left(x^{\mu}, k^{\mu}\right)}{\mathrm{d} \lambda} \\
& =\mathcal{E}_{\mathrm{e}}\left(x^{\mu}, k^{\mu}\right)-\mathcal{A}_{\mathrm{a}}\left(x^{\mu}, k^{\mu}\right) \mathcal{I}\left(x^{\mu}, k^{\mu}\right)-\mathcal{A}_{\mathrm{s}}\left(x^{\mu}, k^{\mu}\right) \mathcal{I}\left(x^{\mu}, k^{\mu}\right) \\
& \quad+\int \mathrm{d} P^{\prime} \mathcal{A}_{\mathrm{s}}\left(x^{\mu}, k^{\prime \mu}\right) \zeta\left(x^{\mu} ; k^{\prime \mu} \rightarrow k^{\mu}\right) \mathcal{I}\left(x^{\mu}, k^{\prime \mu}\right)
\end{aligned}
$$

Here, the coordinate time $t$ is related to the affine parameter $\lambda$ by the relation $k^{t}=\mathrm{d} t / \mathrm{d} \lambda$.

In this study, we solve the RT equation for the case of the coherent and isotropic scattering. We first calculate the total contribution of scattered photons at a point specified by $(i, j)$, where the radiation stress tensor is evaluated. This is done by multiplying the invariant intensities at the points on null geodesics inside a grid by the invariant scattering coefficient $\mathcal{A}_{\mathrm{s}}$ :

$$
\left[\int \mathrm{d} P \mathcal{A}_{\mathrm{s}}\left(x^{\mu}, k^{\mu}\right) \mathcal{I}\left(x^{\mu}, k^{\mu}\right)\right]_{i, j}=\sum_{k=1}^{N_{k}} \mathcal{A}_{\mathrm{s}} \mathcal{I}_{i, j, k} \Delta P_{k},
$$

where $k$ denotes the points inside a grid and $\Delta P_{k}$ is the weight of the momentum integration determined by the energy-conserved scheme described in the previous subsection. This total scattered radiation is distributed isotropically with weight $\Delta P_{k}$ of the momentum integration. That is, the scattered radiation is redistributed to the geodesic coordinates in the nearest neighbour grid. This contribution of incoming scattering photons $\mathcal{E}_{\mathrm{s}}$ is added in the RT equation. In a time-explicit scheme, the RT equation is written as

$\mathcal{I}_{k_{*}}^{i_{*}+1}=\mathcal{I}_{k_{*}}^{i_{*}}+\Delta t\left[\frac{1}{k^{\mathrm{t}}}\left(\mathcal{E}_{\mathrm{e}}+\mathcal{E}_{\mathrm{s}}-\mathcal{A}_{\mathrm{a}} \mathcal{I}-\mathcal{A}_{\mathrm{s}} \mathcal{I}\right)\right]_{k_{*}}^{i_{*}}$.

By solving this equation, we perform the RT simulations incorporating the effects of the absorption and the scattering.

The causality should be preserved in the RT simulations with scattering effects. As denoted in the previous subsection, for one geodesic line, one nearest neighbour specific intensity is used at a grid point where the integration in the momentum space is performed. Along one geodesic line, the contribution from outgoing scattering photons are given from one nearest neighbour geodesic grid and the incoming scattering photons are redistributed at the same nearest neighbour geodesic grid. The scattered photons are treated in the same time and at same spatial position (within the grid zone). With this treatment of scattering photons, the causal RT simulations with scattering effects can be achieved.

Mathematically, the RT equation is an integro-differential equation that involves both derivative and integral of the intensity. In the non-relativistic simulations, the time derivative is dismissed assuming the infinite speed of light. Then, to obtain the self-consistent radiation fields, one should converge the radiation fields by solving the integro-differential equation. Hence, in the past studies, several kinds of iterative methods have been employed to solve the non-relativistic RT equation with scattering. On the other hand, in the causal GR RT in this study, where the travelling time of photon is considered explicitly, such iteration method is not required and the RT equation can be correctly solved with a timeexplicit scheme. This is because in the causal RT simulation, the radiative intensity including the scattering is calculated in temporal order. This is one of the benefits of the causal RT simulations with ARTIST.

\section{GR RT SIMULATIONS WITH ARTIST}

In this section, we present the results of the GR RT simulations performed by ARTIST. To see whether the simulations correctly reflect the GR effects such as light-bending, frame dragging and so on, we compare the results with those by the accurate GR ray-tracing calculations performed by MASTER. Here, we perform the test simulations in the equatorial plane in the Kerr space-time given by the KS coordinates. Since the simulations are done in the equatorial plane, the number of independent components of the radiation stress-energy tensor $R^{\mu \nu}$ is six, that is, $E_{\mathrm{rad}}=R^{\mathrm{tt}}, F^{\mathrm{r}}=R^{\mathrm{tr}}=R^{\mathrm{rt}}$, $F^{\phi}=R^{\mathrm{t} \phi}=R^{\phi \mathrm{t}}, P^{\mathrm{rr}}=R^{\mathrm{rr}}, P^{\phi \phi}=R^{\phi \phi}$, and $P^{\mathrm{r} \phi}=R^{\mathrm{r} \phi}=R^{\phi \mathrm{r}}$. All other components of $R^{\mu v}$ are zero in the simulations. We first report the results of the GRRT simulations without effects of absorption and scattering are done in Sections 3.1-3.4. Then, the simulations with effects of absorption and scattering are reported in Sections 3.5-3.7.

In the following RT simulations, the RT equation is solved in the equatorial plane along the null geodesics with angular resolution of $N_{\phi}=256$ for $0 \leq \phi \leq 2 \pi$. At $\phi=0$ and $2 \pi$, the periodic boundary condition is employed. The radiation stress-energy tensor is calculated in the spherical grid in the equatorial plane. The spatial range of simulations is $0.8 r_{+} \leq r / M \leq 20 r_{+}$with the resolution of $N_{\mathrm{r}}=$ $N_{\phi} / 4$, where $r_{+}$is the radius of the event horizon. The simulation results are shown in the region where $r>r_{+},-12.5 M<x<12.5 M$ and $-12.5 M<y<12.5 M$. Here, $x=r \cos \phi$ and $y=r \sin \phi$. 


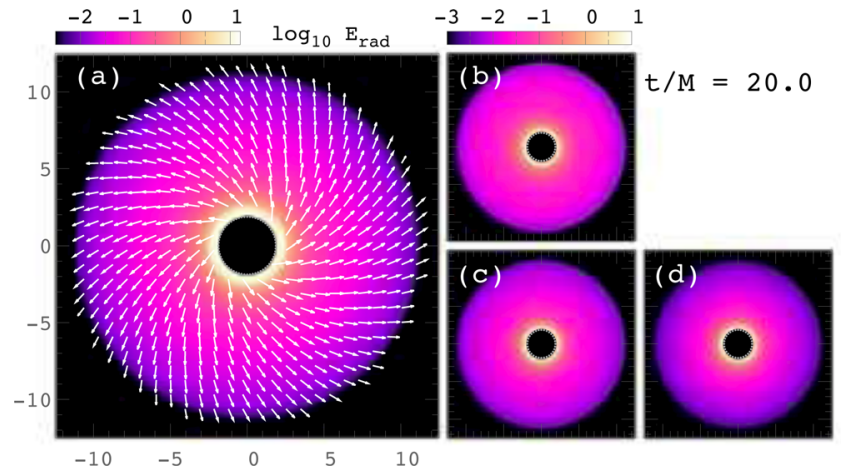

Figure 5. Snapshots at $t / M=20.0$ of the radiation field emitted from an axisymmetric source around a black hole with a spin parameter of $a / M$ $=0.5$. The simulation is performed with double precision. The radiation energy density $E_{\text {rad }}$ are shown in panel (a). The radiation flux vectors $F^{i}$ are shown by arrows. The non-zero components of the radiation stress-tensor $P^{i j}$ are shown in panels (b) $P^{r r}$, (c) $\left|r P^{r \phi}\right|$ and (d) $r^{2} P^{\phi \phi}$. The values of $E_{\mathrm{rad}}$, $P^{r r},\left|r P^{r \phi}\right|$ and $r^{2} P^{\phi \phi}$ are shown in logarithmic scales.

\subsection{Spherical radiation}

We first perform the RT simulation of the radiation emitted from an axisymmetric radiation source in the equatorial plane around a black hole. We assume the source with the isotropic and stationary radiation in the region $r_{+}<r<2.5 \mathrm{M}$. The simulations are performed for two types of numerical accuracy, i.e. single precision and double precision. In these simulations, the axisymmetric radiation field propagates outwards from the vicinity of the horizon. The snapshot of the simulation with the double precision at $t / M=20.0$ is shown in Fig. 5. In the panel (a) of this figure, we plot the values of radiation energy density $E_{\text {rad }}$. The radiation flux vectors $F^{i}$ are shown by arrows. The spatial components of the radiation tensor $P^{i j}$ are shown in the panels (b)-(d). We can see the axisymmetric radiation field in all panels of Fig. 5. The features of the frame-fragging effects can be observed in the plots of the radiation flux vectors.

In order to quantitatively evaluate the results of the simulations, we calculate the fractional errors of the radiation energy along the radial coordinate $r$. The fractional error is calculated as follows. In the simulation, the radiation energy $E_{i, j}$ is calculated for the cell with $i=0,1, \ldots, N_{\mathrm{r}}-1$ and $j=0,1, \ldots, N_{\phi}-1$, where $i$ and $j$ are, respectively, indices for $r$ and $\phi$ coordinates. For each $i$, the maximum fractional errors are calculated as fractional deviations from the average value of the radiation energy, i.e. $\max \left|E_{i, j}-\bar{E}_{i}\right| / \bar{E}_{i}$, where $\bar{E}_{i}=\sum_{j=0}^{N_{\phi}-1} E_{i, j} / N_{\phi}$. In Fig. 6, we show the maximum fractional errors of the radiation energy at $t / M=40.0$ for the simulations with single precision (blue dotted line) and double precision (red solid dotted line). From these results, we can confirm that the radiation energy of the axisymmetric radiation field is precisely calculated by ARTIST within the numerical accuracy used in the simulations.

\subsection{Propagation of photon wavefront}

The propagation of a photon wavefront around a Schwarzschild black hole or a Kerr black hole is originally explored in Hanni (1977). Later on, the wavefront emitted from a source located inside the ergosphere around a Kerr black hole is studied by Takahashi, Ishizuka \& Yokosawa (1990). In these past studies, the BL coordinates are used for the calculations. But, the BL coordinates suffer from the singularity at the event horizon, while the RT can be solved beyond the event horizon in the KS coordinates. Hence, we simulate the wavefront propagation in the KS coordinates.

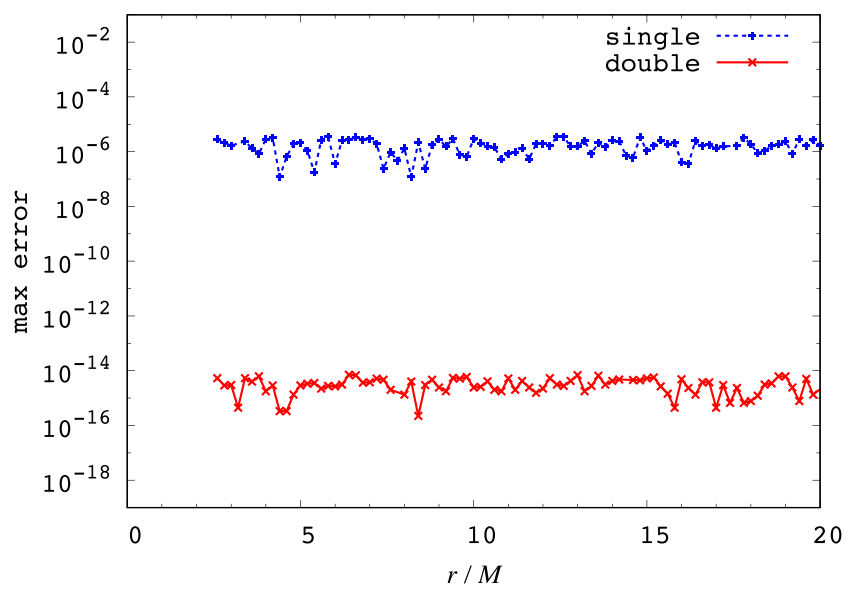

Figure 6. Maximum fractional errors of the radiation energy at $t / M=$ 40.0 of the wavefront simulations performed with single precision (blue dotted line) and double precision (red dotted solid line). The snapshot of the simulation with double precision is shown in Fig. 5.

In these simulations, following five features including the GR effects are tested: (1) the light bending, (2) the accurate propagation with the speed of light, (3) the repeated wavefront collisions around the black hole, (4) the frame-dragging due to black hole rotation and (5) the long-term behaviour reflecting the effects of photon sphere and the black hole rotation. In the wavefront propagation, we can simultaneously check how these physical effects are correctly incorporated in the scheme. Therefore, this is an essential test of the GR RT.

\subsubsection{Results}

In Fig. 7, we present the results of simulations by ARTIST. The Kerr parameter of a BH is assumed to be $a / M=0.5$. A source of photons is initially set at $r=r_{\mathrm{s}}$ and $\phi=\phi_{\mathrm{s}}$ in the equatorial plane. Thus, the Cartesian coordinates of the source are $\left(x_{\mathrm{s}}, y_{\mathrm{s}}\right)=\left(r_{\mathrm{s}} \cos \phi_{\mathrm{s}}, r_{\mathrm{s}} \sin \phi_{\mathrm{s}}\right)$. We assume a Gaussian distribution of the invariant specific intensity for the source. The profile is given by $\mathcal{I}(x, y)=A \exp \left[-d_{\mathrm{s}}^{2} /\left(2 \sigma^{2}\right)\right]$ for the region with $d_{\mathrm{s}} \leq d$, where $d_{\mathrm{s}}=\left[\left(x-x_{\mathrm{s}}\right)^{2}+\left(y-y_{\mathrm{s}}\right)^{2}\right]^{1 / 2}, \sigma$ is the standard deviation for the Gaussian profile for both $x$ - and $y$ directions, $d$ is the cut-off radius of the Gaussian profile and $A$ is the peak value of the Gaussian profile. Here, we assume the following values: $r_{\mathrm{s}} / M=6.0, \phi_{\mathrm{s}}=0.0, \sigma^{2} / M^{2}=1 / 8, d / M=2.0$ and $A=1.0$.

The basic behaviour of each wavefront is expected to be the following: after the emission of photons, a wavefront propagates in curved space-time around a black hole and a part of the wavefront that propagates beyond the event horizon is swallowed by the black hole. Two parts of the wavefront rotate around the black hole in mutually opposite directions. That is, one side rotates in a prograde direction and the other in a retrograde direction. These two wavefronts rotate with different speed owing to the framedragging effects. The wavefront in the prograde direction rotates faster than that in the retrograde direction. These two wavefronts continue to rotate and at some time, the two wavefronts collide with each other. Thereafter, the collisions occur repeatedly and continue eternally. In the present simulations, the wavefront propagation is pursued until $t / M=92.0$, when the wavefront collide with each other five times. By calculating such a long-term behaviour of the photon wavefront, we can check whether the GR effects are correctly solved. 

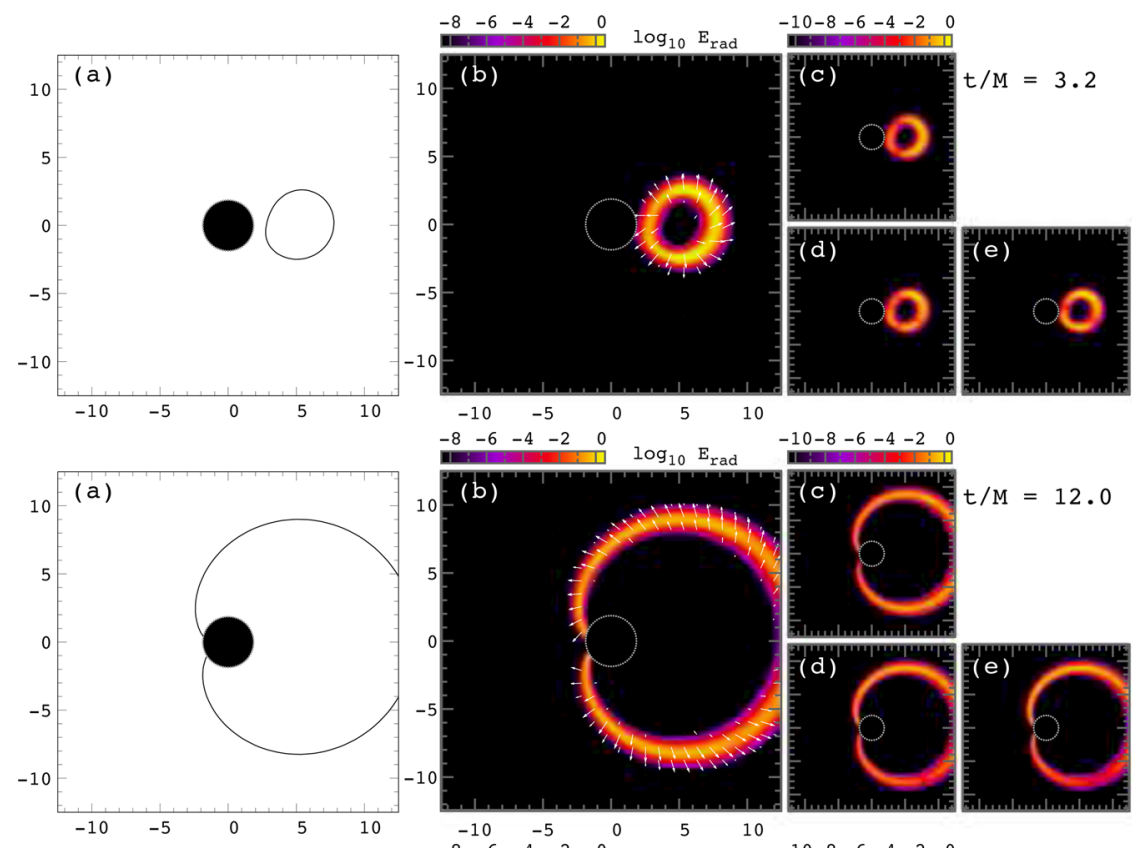

$-10-8-6-4-2 \quad 0$
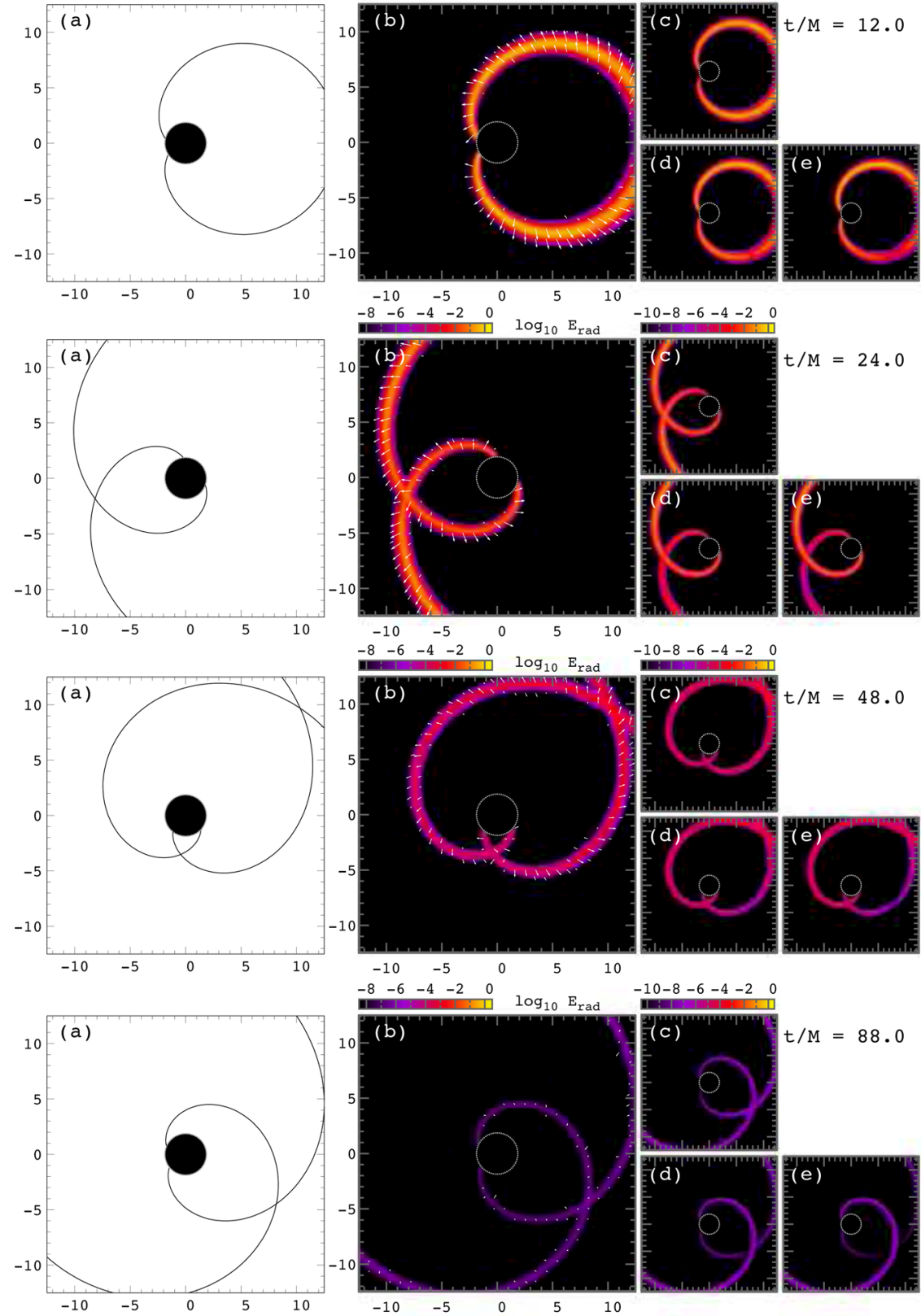

Figure 7. Snapshots at $t / M=3.2,12.0,24.0,48.0$ and 88.0 (from top to bottom) of wavefront propagation around a black hole with a spin parameter of $a / M=0.5$. The exact ray-tracing calculations performed by MASTER are shown in panel (a), where the photon wavefront is plotted by a solid curve and the region of the BH is shown by a filled circle. Panels (b)-(e) show the results by ARTIST. The radiation energy density $E_{\text {rad }}$ are shown in panels (b). The radiation flux vectors $F^{i}$ are shown by arrows. The non-zero components of the radiation stress-tensor $P^{i j}$ are shown in panels (c) $P^{r r}$, (d) $\left|r P^{r \phi}\right|$ and (e) $r^{2} P^{\phi \phi}$. The values of $E_{\mathrm{rad}}, P^{r r},\left|r P^{r \phi}\right|$ and $r^{2} P^{\phi \phi}$ are shown in logarithmic scales. 
Fig. 7 shows the results of simulations at $t / M=3.2,12.0,24.0$, 48.0 and 88.0 (from top to bottom). The panel (a) of Fig. 7 shows the exact ray-tracing calculations performed by MASTER, where the photon wavefront is plotted by a solid curve. The region of the BH is shown by a filled circle. In panels (b)-(e) of Fig. 7, we show the results by ARTIST: (b) the radiation energy density $E_{\text {rad }}$, the spatial components of the radiation stress-energy tensor (c) $P^{\mathrm{rr}}$, (d) $\left|r P^{\mathrm{r} \phi}\right|$ and (e) $r^{2} P^{\phi \phi}$ in logarithmic scales. The component $P^{\mathrm{r} \phi}$ is shown by its absolute value, because this component can be negative at the region when the integral for $k^{\mathrm{r}} k^{\phi}<0$ is dominated. The plot of the radiation energy density is attached by the flux vectors, whose components are calculated from the radiation flux density, $F^{\mathrm{r}}$ and $F^{\phi}$. The lengths of the vectors are arbitrarily normalized by the maximum value.

The snapshot at $t / M=3.2$ shows the results just after the emission. The photon wavefront does not reach the event horizon. The snapshot at $t / M=12.0$ shows the results just before the first collision between one wavefront propagating in the prograde direction and the other in the retrograde direction. The snapshot at $t / M=$ 24.0 shows the results just after the first wavefront collision. At $t / M=48.0$, the second wavefront collision occurs. The snapshot at $t / M=88.0$ shows the wavefront just before five times collisions. In these simulations, we can see that the wavefront propagation by ARTIST is in good agreement with the ray-tracing calculations. This demonstrates that the simulations by our code properly include the relativistic effects of light bending, frame-dragging, and therefore reproduces the long-term behaviour of the wavefront.

Here, we scrutinize the accuracy of ARTIST. The total invariant specific intensity along the photon wavefront within the limited area near a black hole is expected to decrease with time as $\propto e^{-\mathrm{pt}}$, where $t$ is the time from the emission of the wavefront and $p$ is a constant power index. Based on the ray-tracing calculations in Kerr spacetime, we find that the power index $p$ is not dependent upon the initial position of a source and determined solely by the Kerr parameter $a$. This is because in the late phase of the propagation, the behaviour of the wavefront is mainly determined by the null geodesics skimming the photon sphere whose radius is determined by the BH spin and constants of null geodesics. Using this expectation, we can check the accuracy of GR RT calculations around a black hole. Here, we compare the radiation energy as a function of time between the raytracing calculations and the RT simulations by ARTIST. We calculate the total radiation energy $E=R^{00}$ in the regions of $r_{+}<r \leq 20 M$ and $0 \leq \phi<2 \pi$. In Fig. 8, we show the total radiation energy as a function of time. The expectation by the ray-tracing calculations is shown by a dotted straight line that is proportional to $e^{-0.18 t}$. The power index is determined solely by the black hole spin of $a / M=$ 0.5 . The results by ARTIST are shown by a red solid line. In this figure, we can see that the results of ARTIST well reproduce the scaling of the ray-tracing until the time $t / M=90$. For comparison, we also show the results by the simple method (a dashed line), where the integration is done with photon four momentum interpolated from the nearest neighbour data on the null geodesics in equation (6). See Section 2.2 for the simple method. In Fig. 8, we can see that the results by the simple method cannot reproduce the scaling given by the GR ray-tracing calculations. This comparison shows that ARTIST is highly accurate even for long-term wavefront propagation.

\subsubsection{Quantitative analysis of ray effects}

It is known that in the RT simulations, the radiation field emitted from a small radiation source suffers from the so-called ray effects (e.g. Pomraning 1973). That is, for the small radiation source, the

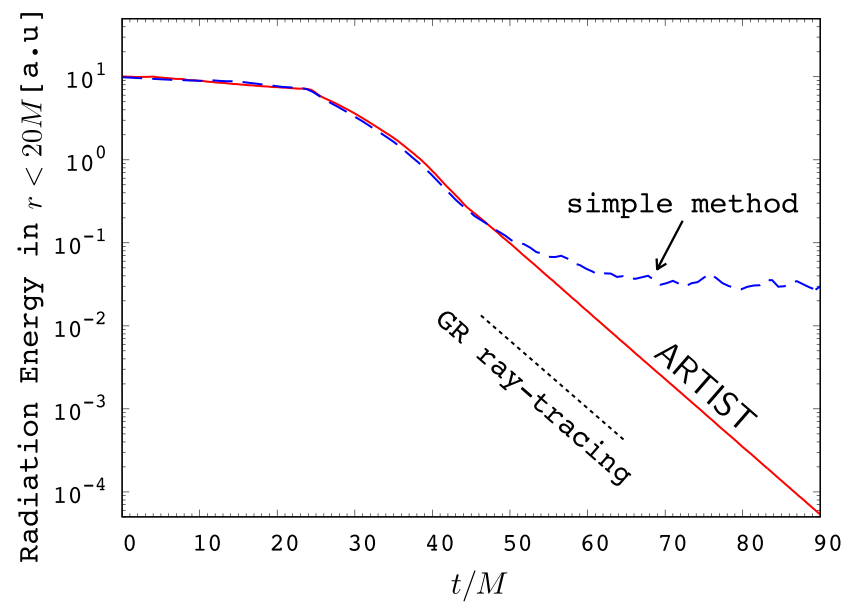

Figure 8. The total radiation energy in the spatial region $r<20 M$ as a function of time in the wavefront shown in Fig. 7. We show the results of ARTIST (a solid line) and the time-dependence by the ray-tracing calculations (a dotted straight line). The results using a simple method, in which the radiation energy is not conserved, is also shown (a dashed line). See the text for the details.

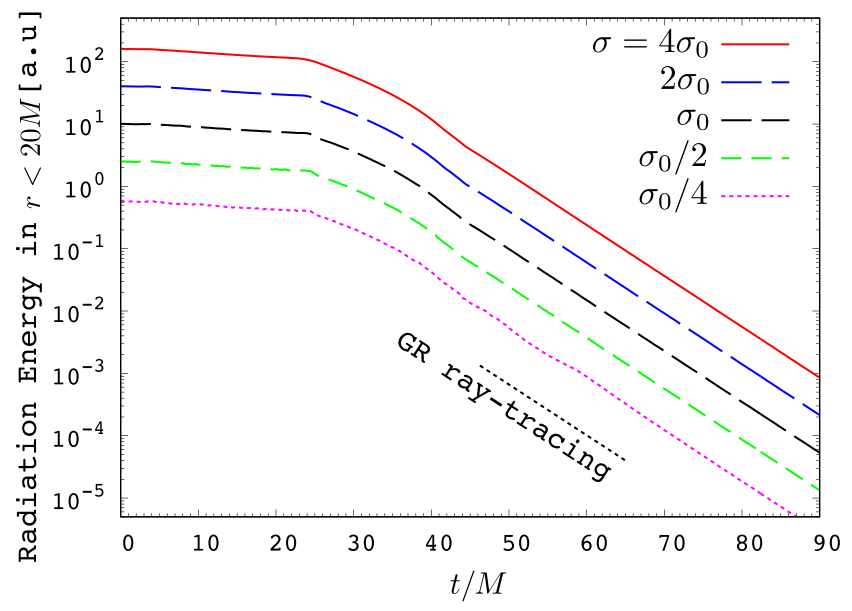

Figure 9. The total radiation energy in the region $r<20 M$ as a function of time in the wavefront simulations for the radiation source (at $t / M=$ $0.0)$ with $\sigma=4 \sigma_{0}, 2 \sigma_{0}, \sigma_{0}, \sigma_{0} / 2$ and $\sigma_{0} / 4$. Here, $\sigma_{0}$ corresponds to the standard deviation of the Gaussian profile of the radiation source in Fig. 8.

radiation energy (and the other components of the radiation tensor) propagating in some directions are lost or decreased. These ray defects are also seen in the RT simulations by SC method. In general, resolving the radiation emitted from a quite small source is difficult in the RT simulations. Therefore, it is important to investigate how correctly the radiation emitted from a small source is calculated by the simulation code.

In order to quantitatively evaluate the influences of ray defects in ARTIST, we perform the wavefront simulations for radiation sources with different sizes. The simulations are performed for a radiation source with $\sigma / \sigma_{0}=1 / 4,1 / 2,1.0,2.0$ and 4.0 , where $\sigma_{0}$ is the standard deviation of the Gaussian profile of the source in the last section. In Fig. 9, we show the radiation energy in $r / M<20.0$ as a function of time for these simulations. We can see that all results reproduce the scaling given by the GR ray-tracing calculations. These results show that ARTIST is accurate for the radiation emitted from a relatively small source. However, in the curve of $\sigma=\sigma_{0} / 4$, we can recognize the slight fluctuations caused by the ray 


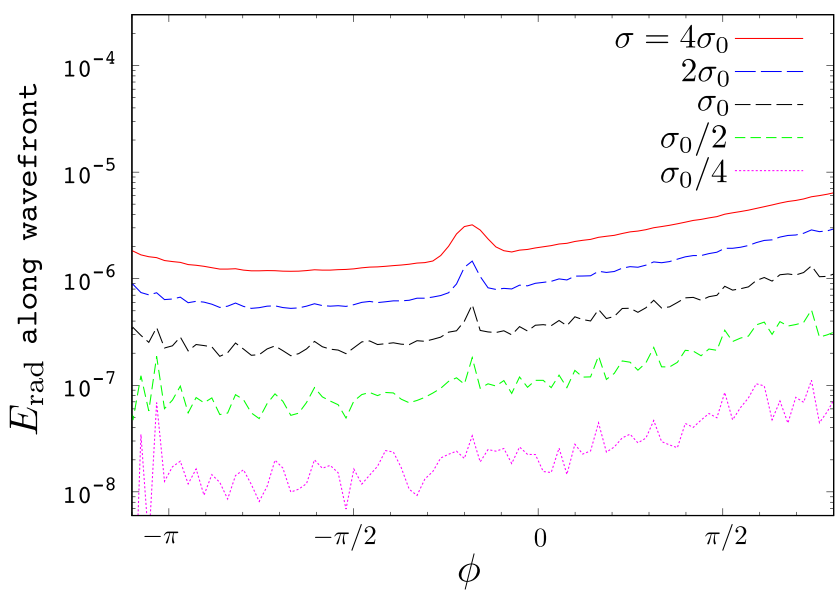

Figure 10. The radiation energy along the wavefront in the simulations in Fig. 9. The results at $t / M=88.0$ are shown.

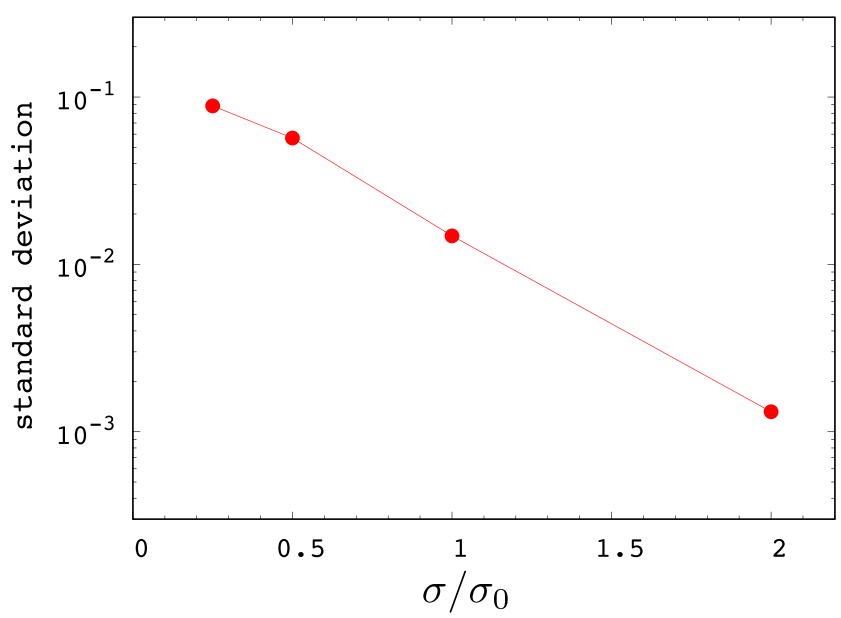

Figure 11. The standard deviation of the fluctuations of the normalized radiation energy along the wavefront shown in Fig. 10 as a function of the source size measured by $\sigma / \sigma_{0}$. The radiation energy is normalized by that for $\sigma=4 \sigma_{0}$

defects. To see the effects of the ray defects more clearly, in Fig. 10, we plot the radiation energy along the wavefront at $t / M=80.0$ for the simulations in Fig. 9. The location of the wavefront where the radiation energy is evaluated is determined by the GR ray-tracing calculation. In this figure, we can see that the fluctuations of the radiation energy are caused by the ray effects and that the amplitude of the fluctuations becomes larger for the smaller source. That is, for a small source, the radiation field does not propagate isotropically even if the isotropic radiation is assumed. In these plots, the bump around $\phi \sim-0.2 \pi$ corresponds to the crossing point of the wavefronts. The quantitative analysis in terms of the ray defects is given in Fig. 11. In this figure, the standard deviation of the fluctuations in the radiation energy in Fig. 10 is plotted as a function of $\sigma$ that determines the source size. In Fig. 11, we use the radiation energy along the wave front which is normalized by the radiation energy with $\sigma=4 \sigma_{0}$. We can confirm that the standard deviation of the fluctuations becomes smaller for larger $\sigma$. From these results, we can expect that ARTIST can resolve the radiation field emitted by a small source if the sufficiently fine geodesic grids are adopted in the RT simulations.

\subsection{Radiation emitted from a luminous wall}

Next, we simulate the trajectories of photons emitted from a luminous wall around a Kerr black hole. We put a stationary wall-like photon source at $x_{\mathrm{s}}=-15 \mathrm{M}$ parallel to $y$-axis in the range $r<20 M$, i.e. $-5 \sqrt{7} M<y<5 \sqrt{7} M$. At the luminous wall, we assume that photons are emitted isotropically. We procure the precise behaviours of wavefronts by ray-tracing calculations and then compare the results to those by ARTIST. We assume the Kerr space-time with the spin parameter $a / M=0.5$. The resolution of geodesic coordinates, the spatial resolution of grids and the spatial range of simulation are the same as those used for the photon wavefront in the previous section. The wavefront propagation is pursued until $t / M=48.0$, at which the radiation fields almost become in a steady state far outside the photon sphere.

In Fig. 12, we show the snapshots of wavefront patterns at $t / M$ $=12.0,28.0$ and 48.0 (top to bottom). The panel (a) shows the results of the ray-tracing calculations performed by MASTER, where the photon wavefront is plotted by solid curves. The region of the $\mathrm{BH}$ is shown by a filled circle. In panels (b)-(e), we show the results by ARTIST in logarithmic scale: (b) the radiation energy density $E_{\mathrm{rad}}$ obtained by ARTIST and the radiation stress-energy tensor, (c) $P^{\mathrm{rr}}$, (d) $\left|r P^{r \phi}\right|$ and (e) $r^{2} P^{\phi \phi}$. The plot of the radiation energy density is attached by the radiation flux vectors, whose components are calculated from the radiation flux density, $F^{\mathrm{r}}$ and $F^{\phi}$.

The snapshot at $t / M=12.0$ shows the results before the wavefront reaches the event horizon. The vectors of the radiation flux density show the directions of the wavefront propagation. The snapshot at $t / M=28.0$ shows the results after passing the BH. In this plot, we can see that the shape of the wavefronts is bent due to the curvature of the space-time.

In addition, we can recognize the features of the frame-dragging. In this case, the black hole is rotating counter-clockwise and the angular velocity of the frame-dragging is proportional to $a / r^{3}$ near the horizon. In the vicinity of the horizon, the radiation flux density along the direction of the frame-dragging is larger than that in the opposite direction. In comparison with the ray-tracing results, we can see that the shape and position of wavefronts at $t / M=12.0$ and 24.0 are in good agreement with the ray-tracing calculations. The snapshot at $t / M=48.0$ shows the radiation fields in a steady state excepting the region near the photon sphere. In the regions where the frame-dragging effects are dominant, the values of $P^{\mathrm{rr}}$ become smaller than those of $P^{\phi \phi}$. This is because $\left|k^{\mathrm{r}}\right| \ll k^{\phi}$ in this region due to the frame-dragging.

\subsection{Radiation emitted by an orbiting hotspot}

In the above, we have shown the test simulations for a static source. Here, as a more realistic and practical problem, we consider a hotspot orbiting a black hole. This problem and the related problems are astrophysically of a great significance and have been explored in the past studies by many authors (e.g. Cunningham \& Bardeen 1972; Bardeen et al. 1972; Cunningham \& Bardeen 1973; Pineault 1977; Pineault \& Roeder 1977; Kapoor 1981; Bao 1992; Karas \& Bao 1992; Stuchlik \& Bao 1992; Bao, Hadrava \& Østgaard 1994; Zakharov 1994; Hollywood et al. 1995; Broderick \& Loeb 2005, 2006; Meyer et al. 2006; Broderick \& Loeb 2009; Li, Kong \& Bambi 2014). For instance, a model of orbiting hotspot is fairly successful in explaining the observed time-variability of the accreting black holes.

Similarly to the previous tests, we obtain the exact patterns of wavefronts by ray-tracing calculations with MASTER. Then, the 

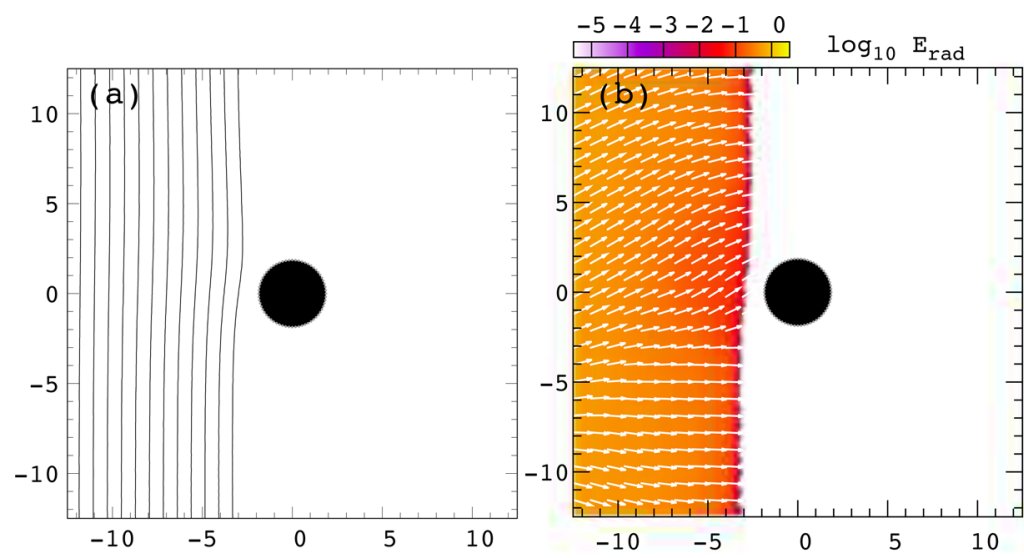

\begin{tabular}{r|r|r|r|}
$-5-4-3-2-1 \quad 0$ \\
\hline $\mid$
\end{tabular}

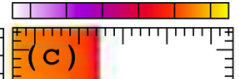

$t / M=12.0$
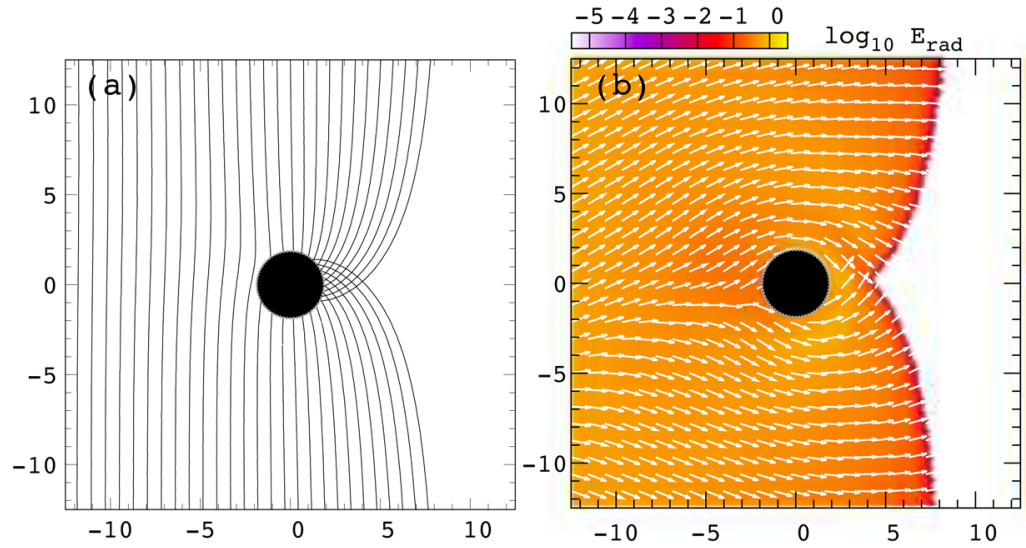

$-5-4-3-2-1 \quad 0$
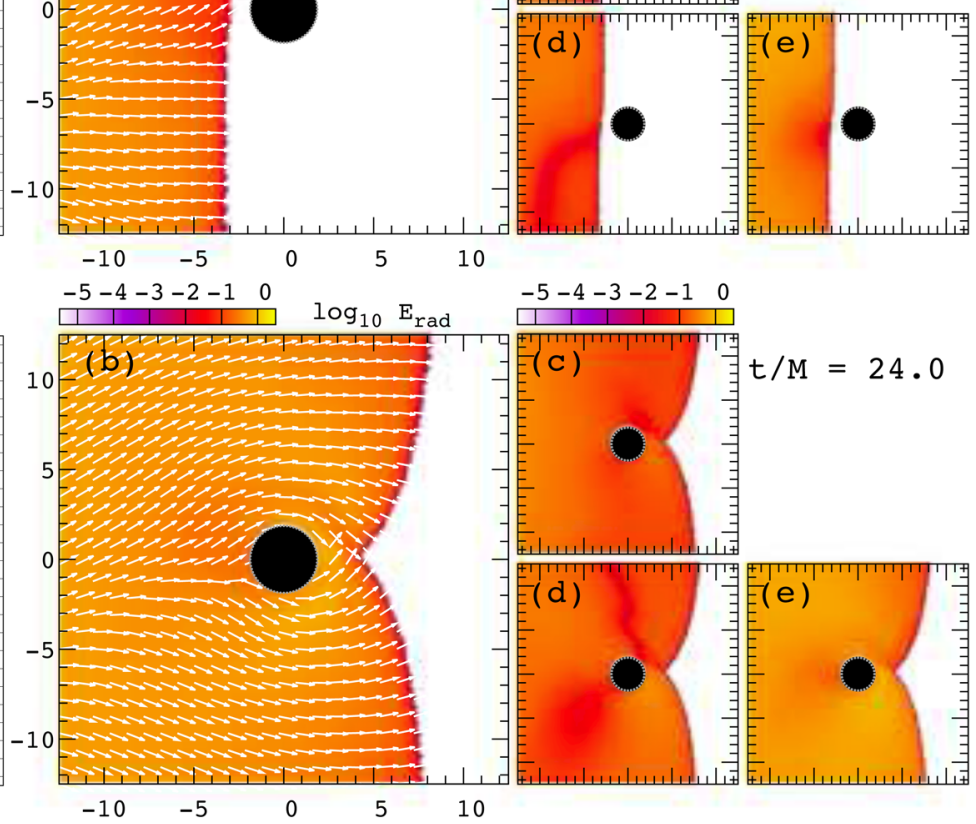

$t / M=24.0$

$\begin{array}{llllll}-1 & 0 & 1 & 2 & \log _{10} & \mathrm{E}_{\mathrm{rad}}\end{array}$
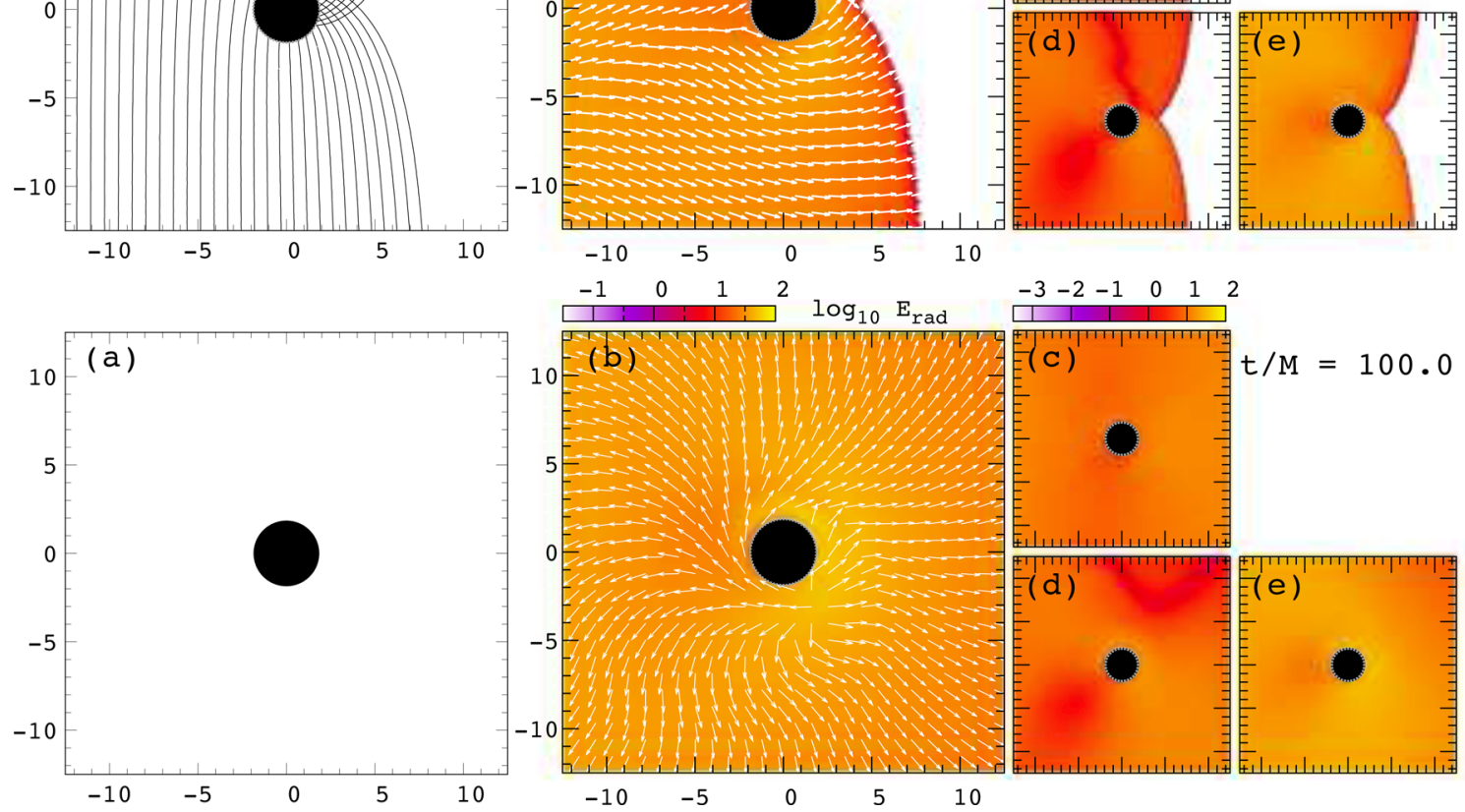

Figure 12. Snapshots of radiation emitted from a luminous wall in Kerr space-time with the spin parameter $a / M=0.5$. The time of each snapshot is $t / M=$ 12.0, 28.0 and 48.0 (from top to bottom). The exact ray-tracing calculations performed by MASTER are shown in panel (a). The radiative transfer calculations with ARTIST are sown in panels (b)-(e). The panel (b) shows the radiation energy density $E_{\text {rad, }}$ where the radiation flux vectors $F^{i}$ are plotted by arrows. The components of radiation stress-tensor $P^{i j}$ are shown in panels (c) $P^{r r}$, (d) $\left|r P^{r \phi}\right|$ and (e) $r^{2} P^{\phi \phi}$ The values of $E_{\mathrm{rad}}, P^{\mathrm{rr}},\left|r P^{r \phi}\right|$ and $r^{2} P^{\mathrm{r} \phi}$ are shown in logarithmic scales. In all the plots, the region of the $\mathrm{BH}$ is shown by the filled circle.

results of RT simulations with ARTIST are compared to ray-tracing calculations. We assume a hotspot orbiting at the innermost stable circular orbit (ISCO) in the Kerr space-time with spin parameter $a / M=0.5$. We adopt the Gaussian profile for the invariant specific intensity of hotspot that is determined by the parameters used in Section 3.2. The resolution of the geodesic coordinates and the number of spatial grids are the same as those used in the photon wavefront simulations presented in Section 3.2. The simulations are performed until $t / M=100.0$, when the radiation fields are almost settled in a steady state.

In Fig. 13, we show snapshots of wavefronts at $t / M=20.0$, 40.0, and 100.0 (from top to bottom), respectively. The panel (a) shows the results of the exact ray-tracing calculations performed by MASTER. The physical quantities shown in the coloured panels are the same as those in Fig. 7. The snapshot at $t / M=20.0$ shows the results after the first collision between the wavefront propagating in the prograde direction and that in retrograde direction. We can see that the spiral structure in the radiation fields begins to form. The shape and the brightness pattern of this structure depends on the Kerr parameter. At $t / M=40.0$, the spiral structure continues to rotate around the black hole. At $t / M=100.0$, the radiation fields are almost settled in a steady state. At this stage, the spiral structure consists of wavefronts emitted at different time. In these simulations, we can see that the structures of the radiation fields 

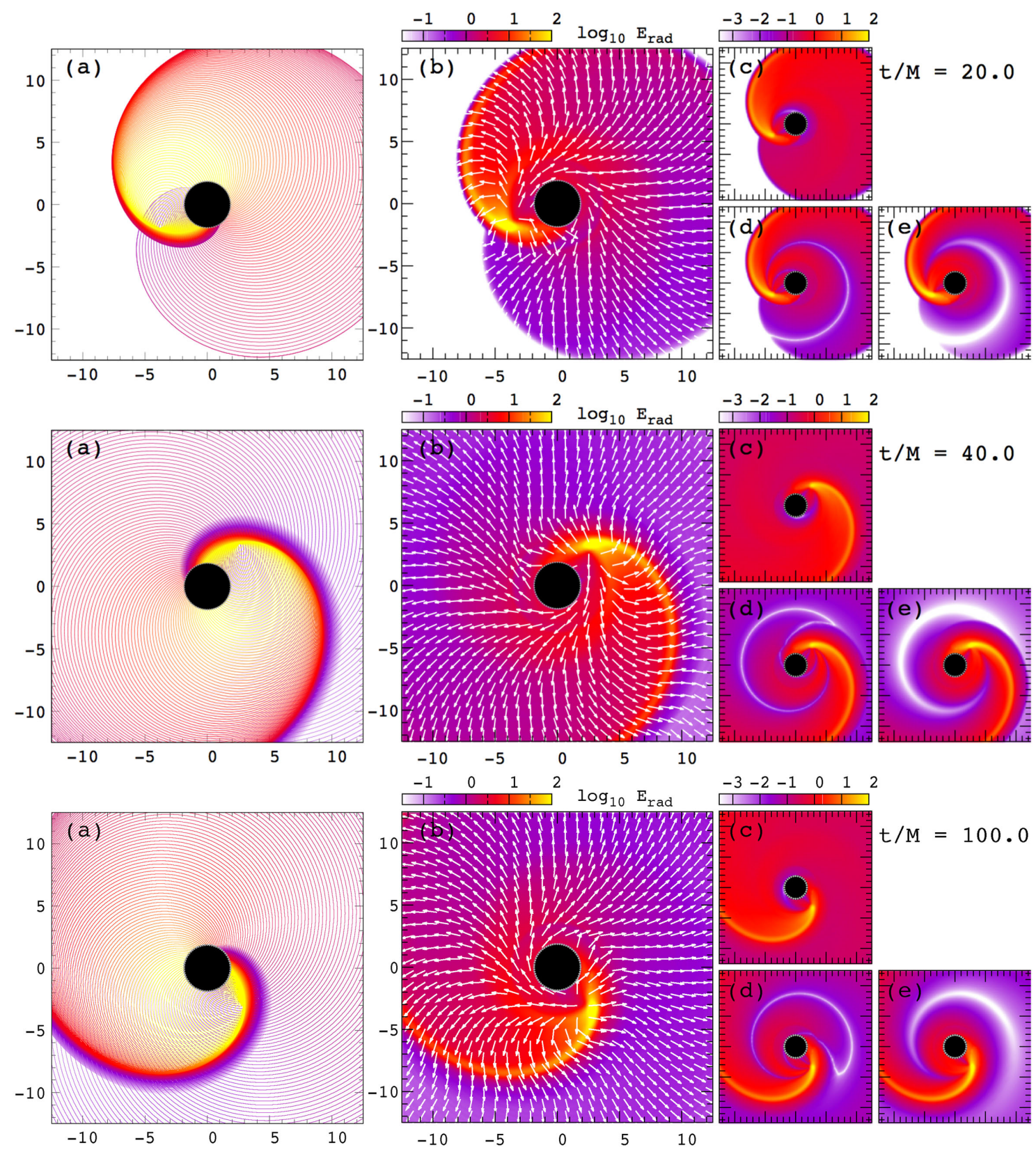

Figure 13. Snapshots at $t / M=20.0,40.0$ and 100.0 (from top to bottom) of the radiation emitted from a hotspot orbiting a black hole with the spin parameter $a / M=0.5$. The hotspot is moving in a prograde orbit at the ISCO. The ray-tracing calculations performed by MASTER are shown in panel (a), where the wavefront is plotted by a solid curve. Panels (b)-(e) show the results by ARTIST. The radiation energy density $E_{\text {rad }}$ is shown in panel (b). The radiation flux vectors $F^{i}$ are shown by arrows. The non-zero components of the radiation stress-tensor $P^{i j}$ are shown in panels (c)-(e). That is, we show (c) $P^{r r}$, (d) $\left|r P^{r \phi}\right|$ and (e) $r^{2} P^{\phi \phi}$. These values are shown in logarithmic scales. In all the plots, the region of the BH is shown by the filled circle.

are quite similar to those given by the ray-tracing calculations. This shows that ARTIST can correctly solve the RT for a moving source.

\subsection{Propagation of photon wavefront with effects of scattering and absorption}

In this subsection (Section 3.5) and the subsequent subsections (Sections 3.6 and 3.7), the effects of scattering and absorption are included in the RT simulations with ARTIST. In this study, we only consider the case of elastic and isotropic scattering in the LNRF.

In the present subsection, we calculate the same problem in Section 3.2 but with effects of scattering and absorption. These ef- fects are included based on the calculation method described in Section 2.3. In this problem, the main part of the wavefront propagates in a similar manner as Section 3.2 and a part of photons emitted from the wavefront are absorbed and scattered in all regions.

\subsubsection{Results}

We first simulate the problem only with scattering. In Fig. 14, we show the results of wavefront problem with effects of (A) scattering (left-hand side) and (B) absorption (right-hand side). In these simulations, we assume $\varpi=1$ for the result (A) and $\varpi=0$ for the result (B). For both simulations, $\mathcal{A}=0.001$ is 
(A) results with scattering
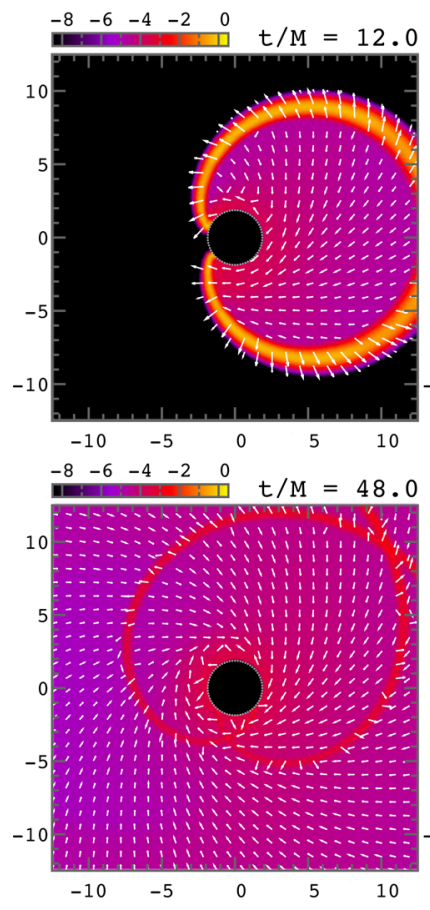

$\begin{array}{lllll}-8 & -6 & -4 & -2 & 0\end{array}$
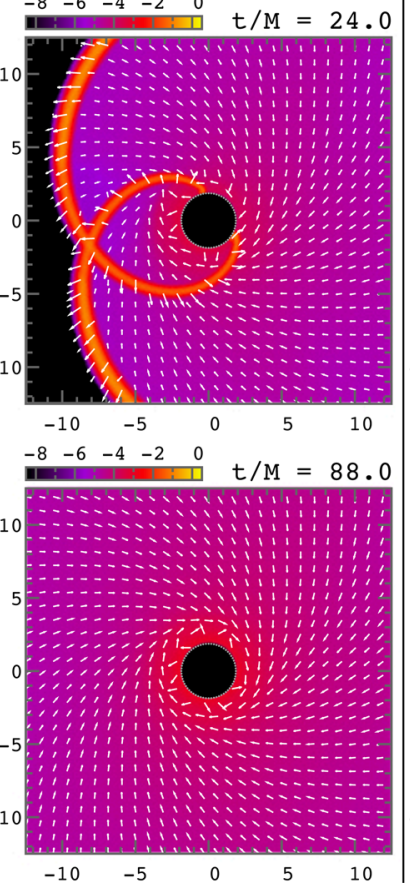

(B) results with absorption
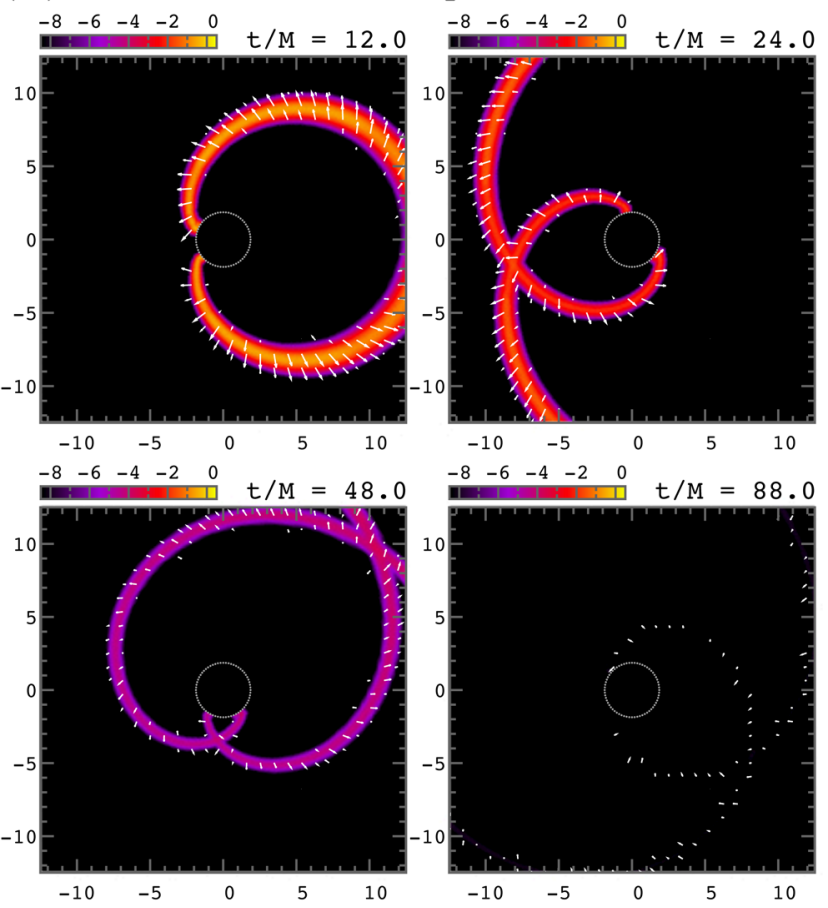

Figure 14. Same as Fig. 7 but with effects of (A) scattering (left-hand side) and (B) absorption (right-hand side). Snapshots at $t / M=12.0,24.0,48.0$ and 88.0 are shown for both results (A) and (B).

assumed. In this figure, snapshots at $t / M=12.0,24.0,48.0$ and 88.0 are shown.

In the result (A) of Fig. 14, the snapshot at $t / M=12.0$ shows the wavefront before the first collision of wavefronts (cf. Section 3.2). At this snapshot, we can see that the wavefront forms the ring-like shape and the scattered photons are distributed inside the ring. A part of the scattered photons and the wavefront are swallowed by the black hole. The snapshot at $t / M=24.0$ shows the wavefront just after the first collision. After the first collision of the unperturbed wavefronts, they begin to propagate among the scattered photons. The radiation energy of the unperturbed wavefront near the black hole continues to decrease as seen in Section 3.2, but in the present case, the scattered photons exist everywhere among the unperturbed wavefront. This feature can be seen in the snapshot at $t / M=48.0$. Finally, the unperturbed wavefront completely disappears in the scattered photons as shown in the snapshot at $t / M=88.0$. After this phase, the black hole swallows the scattered photons continuously but very slowly. With effects of the scattering, the radiation energy decrease and the spatial distribution of the radiation is smoothed, i.e. the ring-like shape of the wavefront disappear.

In the result (B) of Fig. 14, the results with absorption are shown with the colour-bar range used in the plots of the result (A) (and Fig. 7). In this simulation, we can see that the radiation energy decrease more rapidly than the result in Fig. 7. The shape of the wavefront is same as the results of GR ray-tracing calculations shown in Fig. 7. At the snapshot at $t / M=88.0$, the wavefront cannot be shown with the colour-bar range used in Fig. 14.

In Fig. 15, the total radiation energy inside the region of $r<20 M$ is shown as a function of time $t / M$. This figure can be compared with Fig. 8. In this figure, the result without scattering is shown by a red solid line and this is the same result shown in Fig. 8. The result with scattering in Fig. 14 is shown by a blue dashed line. In this simulation, we can see two phases: wavefront-dominant phase and

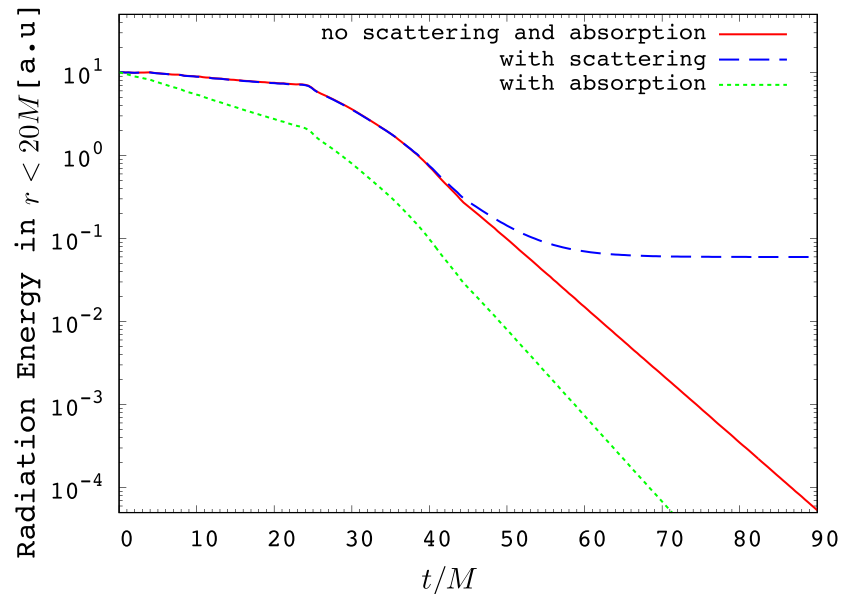

Figure 15. The total radiation energy in the spatial region $r<20 M$ as a function of time in the wavefront simulations shown in Figs 7 (a red solid line) and 14 (a blue dashed line for A in Fig. 14, a green dotted line for B in Fig. 14).

scattering photon-dominant phase. Until the time $t / M \sim 50.0$, the results without scattering (red solid line) and with scattering (blue dashed line) are quite similar. That is, until this time, the contribution of the wavefront is dominated in the RT simulation with scattering. In addition, in the wavefront-dominant phase (i.e. until the time $t / M \sim 50.0$ ), we can see the total radiation energy for the case with scattering is slightly smaller than the case without scattering. This is because a part of the scattered photons are swallowed by the black hole. After the time $t / M \sim 50.0$, as seen in Fig. 14, the wavefront disappears in scattering photons, i.e. the contribution of the scattered photons begins to dominate. After this time, the total radiation energy due to the scattered photons decreases quite slowly. 


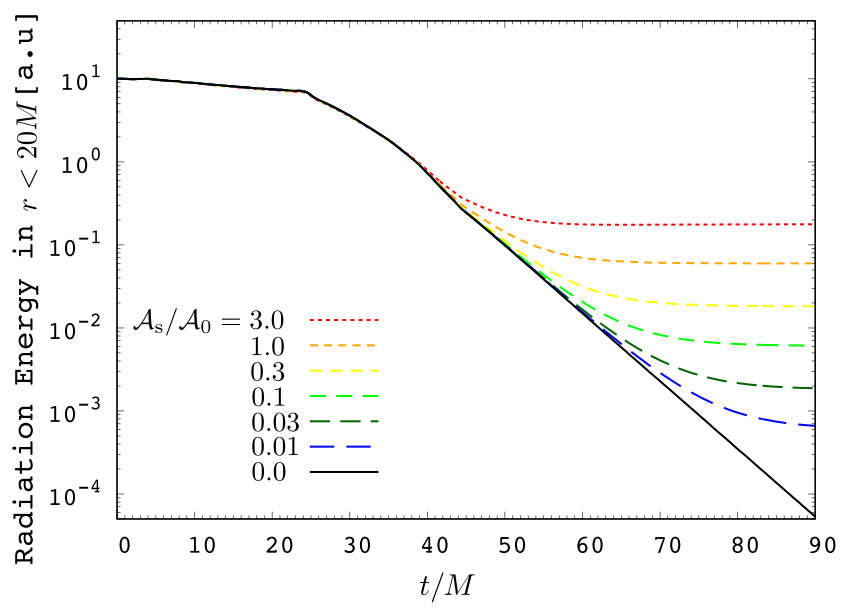

Figure 16. Same as Fig. 15 but for $\mathcal{A}_{\mathrm{s}} / \mathcal{A}_{0}=0.0,0.01,0.03,0.1,0.3,1.0$ and 3.0, where $\mathcal{A}_{0}$ are the scattering coefficients used in Fig. 15.

In Fig. 15, we also show the results with absorption in Fig. 14 by a green dotted line. In this case, the total radiation energy in the region $r<20 M$ continue to decrease from $t / M=0$. As we can expect, the radiation energy of the wavefront decrease more quickly than the case of no scattering and absorption.

\subsubsection{Quantitative analysis of scattering}

The signatures of the scattering in RT simulations are controlled by a scattering coefficient $\mathcal{A}_{\mathrm{s}}$. For giving the quantitative analysis in terms of scattering in ARTIST, the wavefront simulations for several values of the scattering coefficient are performed. As explained in the last section, in the wavefront simulation, while the early phase of the simulation represents the wavefront-dominant phase, the late phase represents the scattering-dominant phase. Therefore, we can expect that the amount of the radiation energy in the scattering-dominant phase is determined by the scattering coefficient. In Fig. 16, the radiation energy in $r<20 M$ in the RT simulation is shown as a function of time for the scattering coefficients $\mathcal{A}_{\mathrm{s}} / \mathcal{A}_{0}=0.0,0.01,0.03,0.1,0.3,1.0$ and 3.0. We can see that the amount of the radiation energy at the late phase become larger for the larger values of the scattering coefficient. This feature can be quantitatively shown in Fig. 17. In this figure, the amount of the radiation energy at $t / M=92.0$, i.e. the scattering-dominant phase, is shown as a function of the scattering coefficient for all the simulations. In this plot, since the inclination of the line shown in logarithmic scale is approximately unity, we can recognize that the amount of the radiation energy mainly due to the scattering photons are roughly proportional to the scattering coefficient.

\subsection{Radiation emitted from a luminous wall with effects of scattering and absorption}

As a next RT simulation with scattering and absorption, we calculate the radiation field emitted from a luminous wall (same as Section 3.3) that propagates in the region with a perfect absorber near the black hole. We consider the perfect absorber located at $x=$ $5.0 M$ and $-10 M<y<10 M$. We assume that the specific intensity is completely absorbed at the perfect absorber. Fig. 18 shows the results of the RT simulation by ARTIST for the cases (A) without scattering (left) and (B) with scattering (right). Snapshots at $t / M$ $=28.0,40.0$ and 80.0 are shown in Fig. 18. In the simulation

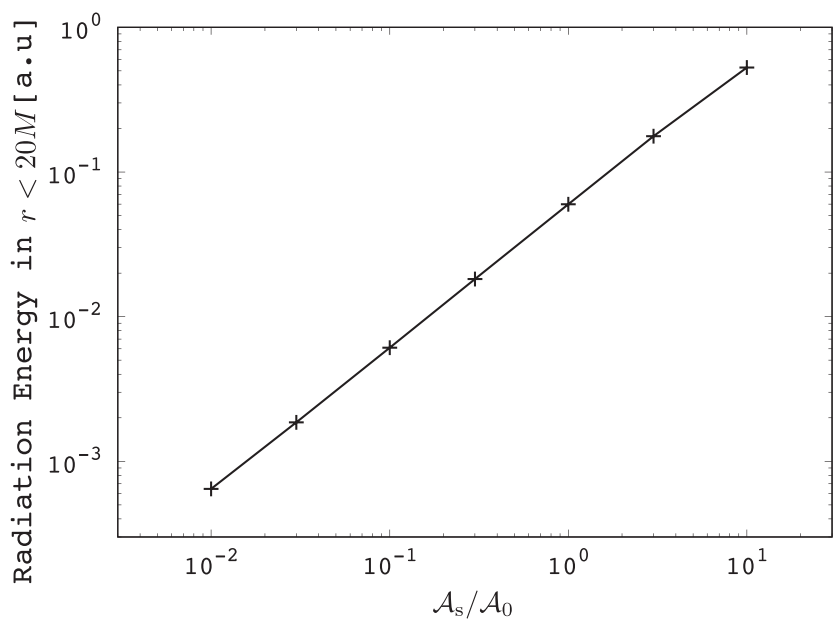

Figure 17. The radiation energy in $r<20 M$ at $t / M=92.0$ as a function of $\mathcal{A}_{\mathrm{s}} / \mathcal{A}_{0}$ in Fig. 16.

without scattering, shown in the panels in the left-hand side of Fig. 18, we can see no radiation behind the perfect absorber. The shape of the region with no radiation is determined by the null geodesics passing at the edge of the perfect absorber. The radiation propagation is pursued until $t / M=80.0$, at which the radiation fields almost become settled in a steady state outside the photon sphere.

In addition, we perform the simulation with scattering, the results of which are shown in the panels in the right-hand side of Fig. 18. In this simulation, we assume $\varpi=1$ and $\mathcal{A}=0.001$. The scattered photons gradually sneak into the region behind the perfect absorber as shown in the snapshots at $t / M=28.0$ and 40.0 in Fig. 18. This simulation is also performed until the radiation fields become almost in a steady state far outside the photon sphere. In the snapshot at $t / M=80.0$, we can see the region behind the perfect absorber is filled with the scattered photons.

\subsection{Radiation emitted by an orbiting hotspot with effects of scattering and absorption}

As a final RT simulation with ARTIST, we simulate the radiation field emitted from the hotspot with effects of scattering and absorption. We calculate the same problem in Section 3.4 but with absorption and scattering. For absorption and scattering, we assume $\varpi=0.5$ and $\mathcal{A}=0.01$. In Fig. 19, the snapshots at $t / M=20.0,40.0$ and 80.0 (top to bottom) are shown. In this simulation, the radiation emitted from the hotspot is absorbed along the geodesics. Therefore, the radiation energy decreases more rapidly than the case in Section 13. In addition, we can see that the radiation field is slightly smeared out by the scattering effects. We pursue this simulation until $t / M=80.0$, at which the radiation fields become in the steady state.

\section{CONCLUSIONS}

In this study, we develop the RT code ARTIST that solves the dynamical radiation field around the Kerr black hole. In the course of the development, the GR ray-tracing code MASTER is also developed. ARTIST is developed based on the ART method in the cosmological simulations under Newtonian gravity so as to include the GR effects of the radiation. In the ARTIST, the time-dependent RT equation is directly solved with time-explicit differencing and 
(A) results without scattering
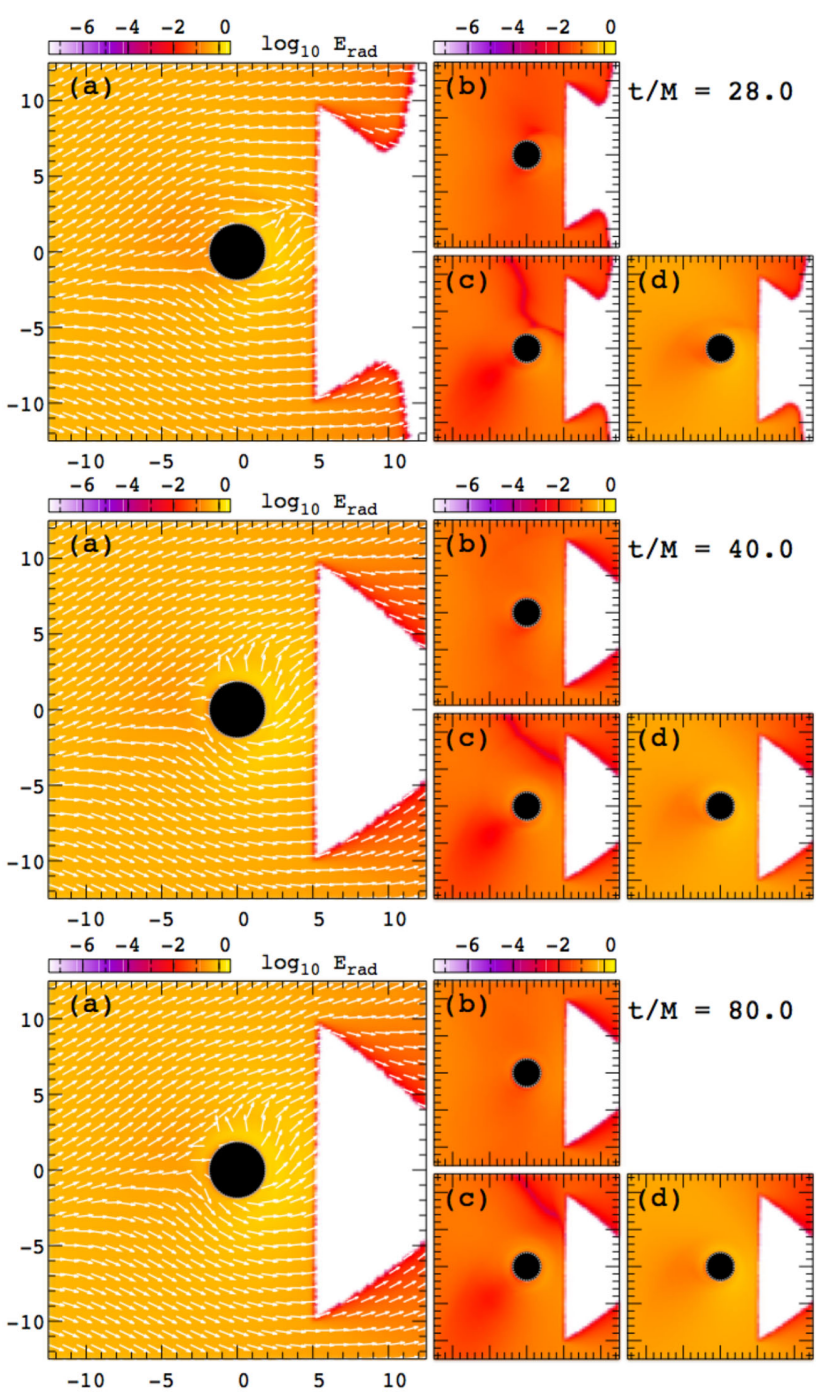

\section{(B) results with scattering}
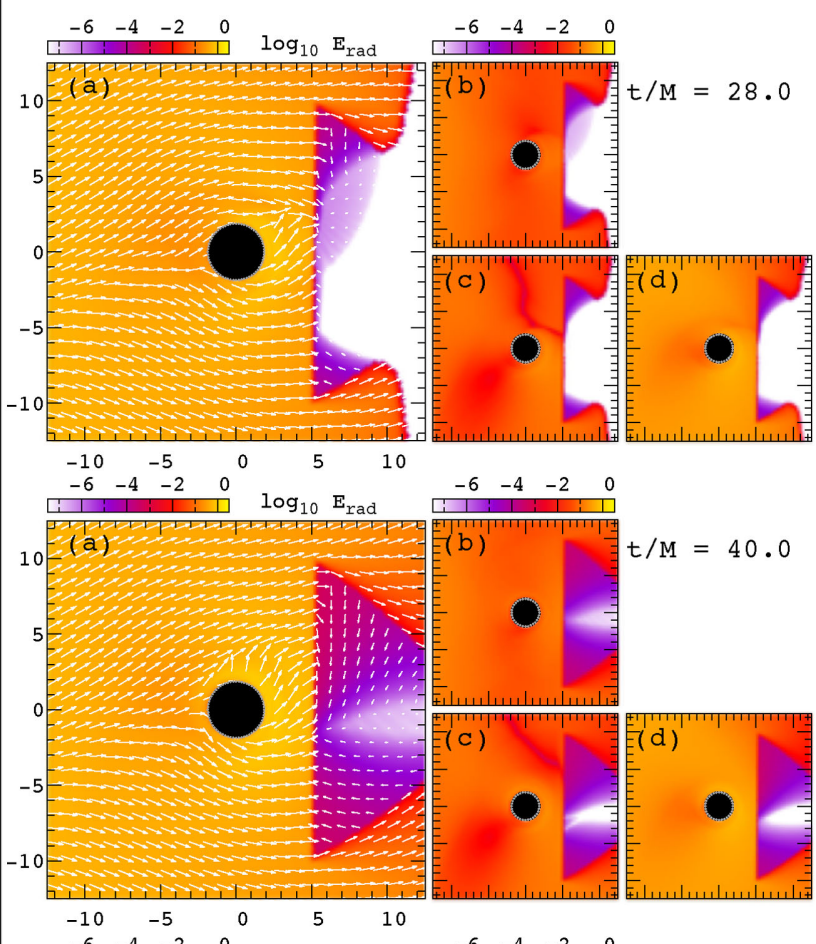

$\begin{array}{llll}-6 & -4 & -2 & 0\end{array}$
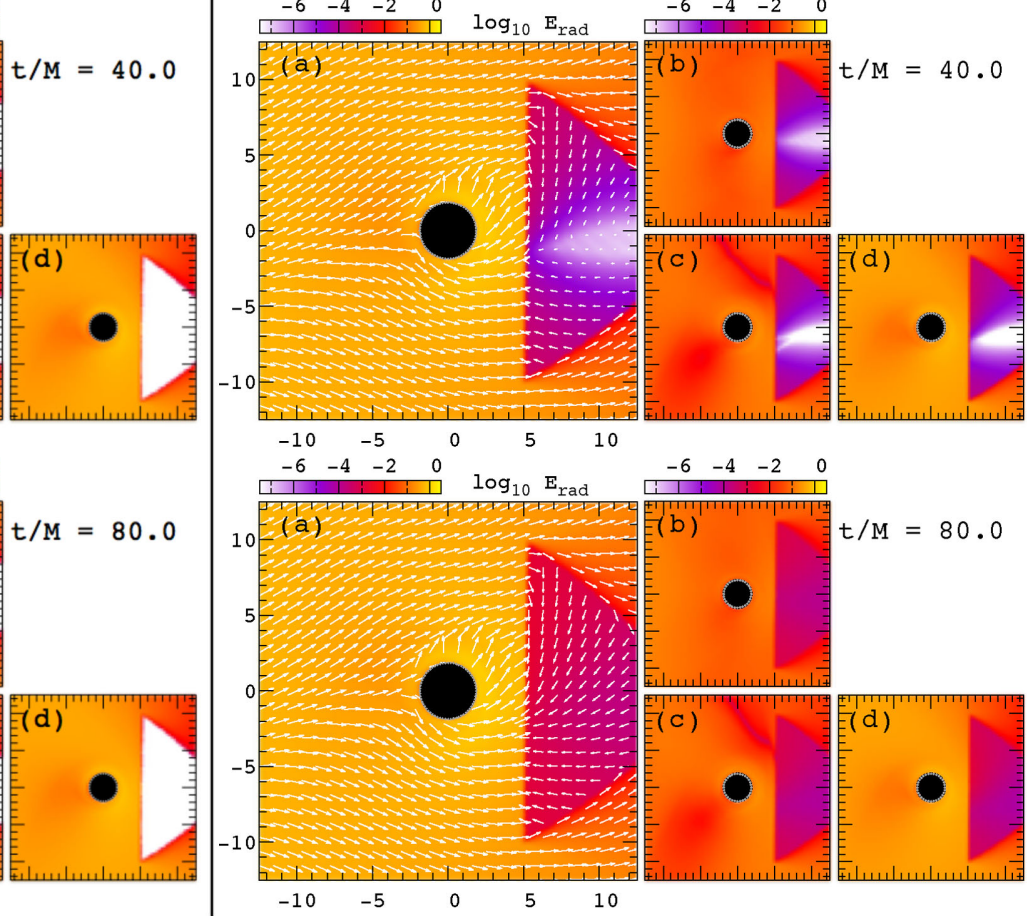

Figure 18. Same as Fig. 12 but with a perfect absorber located at $x=5 M$ and $-10 M<y<10 M$. The time of each snapshot is $t / M=28.0,40.0$ and 80.0 (from top to bottom). We show the results of the simulations (A) without scattering (left) and (B) with scattering (right).

the invariant intensity is calculated in the phase space of the radiation. In order to do this, the grids along the null geodesics are set in the phase space (see Section 2.1) and the RT equation is solved along the null geodesics (see Section 2.3). In the calculations of the radiation tensor and the scattering term of the RT equation, the integration in the momentum space is performed with the energy-conserved scheme in Section 2.2. We can confirm that ARTIST can correctly solve the radiation energy throughout the RT simulation (see Fig. 8). In ARTIST, the causal RT simulation can be performed by solving the RT equation along the null geodesics in the Kerr space-time. Therefore, ARTIST can simulate the radiation field both in the optically thin and thick regions. In the optically thin region, ARTIST can basically reproduce the results of the GR ray-tracing.

With ARTIST, we solve the problems in the Kerr space-time and a part of the results is compared with those of the GR raytracing by MASTER. The RT simulations with ARTIST are reported in Section 3. We first solve the spherical radiation emitted from the vicinity of the black hole horizon (Section 3.1 ). Then, the propaga- tion of the wavefront emitted near the rotating black hole is simulated (Section 3.2). By this test, we can check how correctly ARTIST solve the radiation field with the physical effects such as (1) the light bending, (2) the propagation with the speed of light, (3) the repeated radiation collisions around the black hole, (4) the frame-dragging effects due to black hole's rotation and (5) the long-term behaviour reflecting the effects of photon sphere and the black hole's rotation. We can confirm that the results of ARTIST well reproduce the results of ray-tracing calculations including the late-time behaviours (see Figs 7 and 8). In these tests, we give the quantitative analysis of the ray defects of the radiation field and we can confirm that even in the case that the signatures of the ray defects are seen in the simulations, the total radiation energy is correctly calculated, i.e. the GR ray-tracing results are reproduced in the late phase of the simulation. We also solve the radiation fields emitted from the photon wall (Section 3.3) and the hotspot rotating around the black hole (Section 3.4). In these tests, ARTIST also reproduce the results of the GR ray-tracing. Judging from the results of these three tests, we can consider that ARTIST can correctly solve the radiation field around 

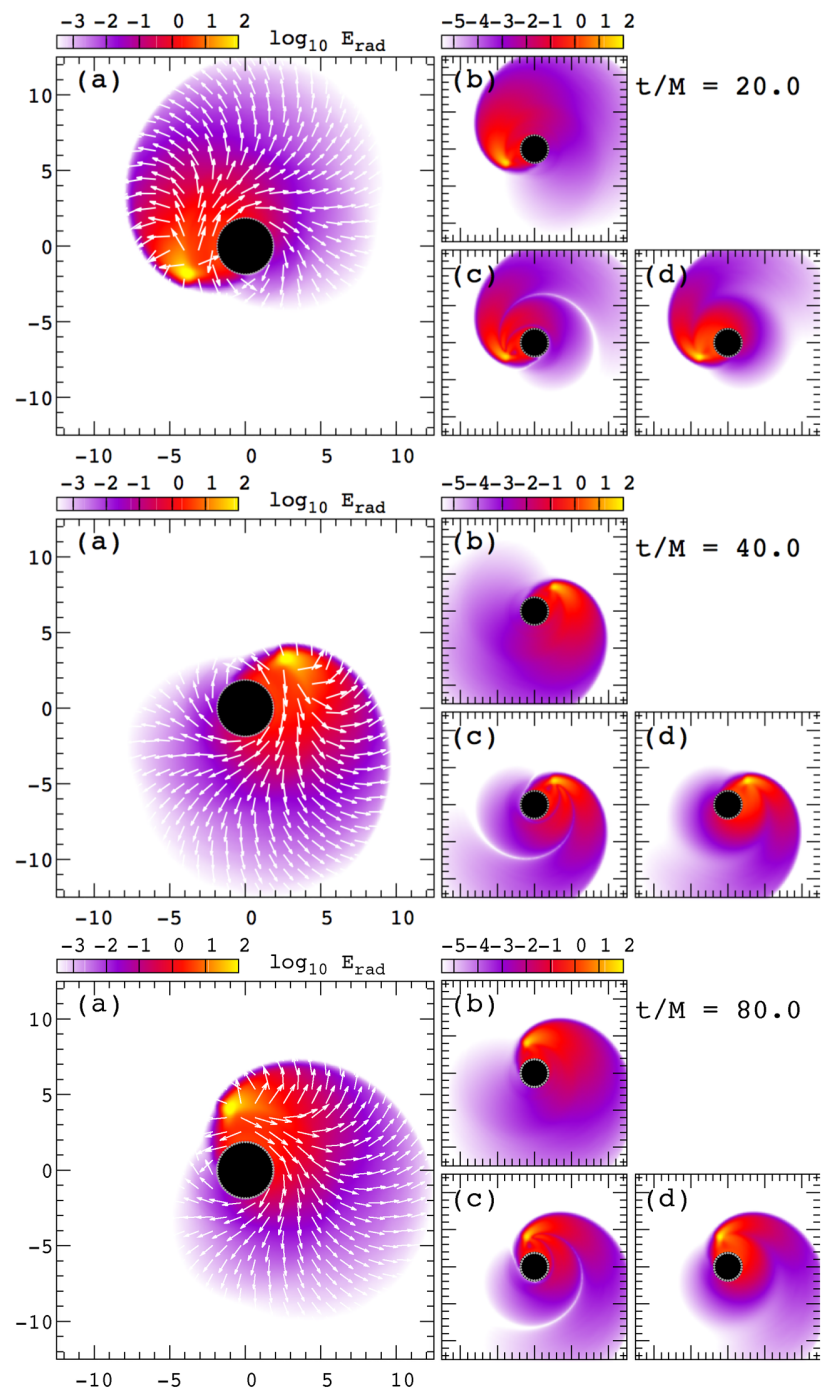

Figure 19. Same as Fig. 13 but with effects of absorption and scattering of radiation. The snapshots at $t / M=20.0,40.0$ and 80.0 (top to bottom) are shown.

the Kerr black hole. It is noted that although all the simulations in this study are performed in the equatorial plane around a Kerr black hole, the methods in ARTIST can be applied to three-dimensional simulations.

With ARTIST, we also solve the radiation field with effects of scattering and absorption. In the simulations with scattering, we take into account both the outgoing and incoming scattering photons. The scattering effect is included to preserve the causality (see Section 2.3). With effects of scattering and absorption, we solve the problems of the wavefront propagation (Section 3.5) and the radiation fields emitted from the luminous wall (Section 3.6) and the hotspot (Section 3.7). Judging from the shapes of the wavefront (Fig. 14) and the region with no radiation behind the perfect absorber (Fig. 18), in these tests, we obtain the reasonable results in which we can expect that the GR effects are correctly included. In the wavefront simulations, we give the quantitative analysis of scattering and we confirm that the amount of the radiation energy in the scattering phase, i.e. the late phase, of the simulation is proportional to the invariant absorption coefficient.

Finally, it should be noted that the additional analysis and possibly some modifications of ARTIST in terms of the treatment of repeated scattering would also be required in future works. In the RT simulations in curved space-time, the simulations should reproduce both the GR ray-tracing results in the optically thin limit and some results in the optical thick limit. In the optically thick case, when the fractional energy transfer per scattering is small, some analytic solutions of the Kompaneets equation are known for non-relativistic case (e.g. Sunyaev \& Titarchuk 1980). These solutions include the case that the diffusion time $\Delta t_{\mathrm{D}}$ of a scattered photon is quite small, i.e. $\Delta t_{\mathrm{D}} \ll 1$. In order to handle the scattering photons with $\Delta t_{\mathrm{D}} \ll$ 1 by ARTIST, the geodesic coordinate with $\Delta t<\Delta t_{\mathrm{D}} \ll 1$ is required. The time-scale of the scattering process could be an atomic scale and it is practically impossible to perform the RT simulations in such time-scale. Therefore, we consider that the valid treatment of the scattering photons with $\Delta t_{\mathrm{D}} \ll 1$ in the RT simulations in curved space-time is quite important topic for future studies. In addition, by coupling ARTIST with GRHD/GRMHD solver, we expect that the causal GRRHD/GRRMHD simulations can be achieved in future.

\section{ACKNOWLEDGEMENTS}

We would like to thank the anonymous reviewer for invaluable comments and useful suggestions. The authors are grateful to K. Ohsuga, H. Takahashi, M. Shibata, Y. Sekiguchi, S. Mineshige, R. Matsumoto, N. Kawakatsu, K. Konno, T. Nagasawa, K. Kumazaki and K. Sumiyoshi for valuable discussions. This work was supported in part by a Grant-in-Aid for Challenging Exploratory Research of JSPS, 25610044 (RT), by Research Project for Young Researcher of National Institute of Technology, H27-Young-No.8 (RT), by a Grant-in-Aid for Scientific Research (C) of JSPS, 16K05302 (RT) and by a Grant-in-Aid for Scientific Research (B) of JSPS, 15H03638 (MU). This research was also supported in part by Interdisciplinary Computational Science Program in Center for Computational Sciences, University of Tsukuba.

\section{REFERENCES}

Abdikamalov E., Burrows A., Ott C. D., Löffler F., O’Connor E., Dolence J. C., Schnetter E., 2012, ApJ, ApJ, 755, 111

Abel T., Norman M. L., Madau P., 1999, ApJ, 523, 66

Abramowicz M. A., Fragile P. C., 2013, Living Rev. Relativ., 16, 1

Abramowicz M. A., Czerny B., Lasota J. P., Szuszkiewicz E., 1988, ApJ, 332,646

Balbus S. A., Hawley J. F., 1991, ApJ, 376, 214

Balbus S. A., Hawley J. F., 1998, Rev. Mod. Phys., 70, 1

Bao G., 1992, A\&A, 257, 594

Bao G., Hadrava P., Østgaard E., 1994, ApJ, 425, 63

Bardeen J. M., 1973, in DeWittB. S., DeWitt C., eds, Black holes (Les astres occlus). Gordon and Breach, New York, p. 215

Bardeen J. M., Press W. H., Teukolsky S. A., 1972, ApJ, 178, 347

Beckwith K., Done C., 2005, MNRAS, 359, 1217

Begelman M. C., 1978, MNRAS, 184, 53

Blandford R. D., Begelman M. C., 1999, MNRAS, 303, L1

Broderick A. E., Loeb A., 2005, MNRAS, 363, 353

Broderick A. E., Loeb A., 2006, MNRAS, 367, 905

Broderick A. E., Loeb A., 2006, J. Phys.: Conf. Ser., 54, 448

Broderick A. E., Loeb A., 2009, Sci. Am., 301, 42

Bromley B. C., Chen K., Miller W. A., 1997, ApJ, 475, 57

Čadež A., Kostić U., 2005, Phys. Rev. D, 72, 104024

Cardall C. Y., Lentz E. J., Mezzacappa A., 2005, Phys. Rev. D, 72, 043007

Cardall C. Y., Endeve E., Mezzacappa A., 2013, Phys. Rev. D, 88, 023011

Castor J. I., 2004, Radiation Hydrodynamics. Cambridge Univ. Press, Cambridge

Chandrasekhar S., 1950, Radiative Transfer. Clarendon Press, Oxford

Ciardi B., Ferrara A., Marri S., Raimondo G., 2001, MNRAS, 324, 381 
Cunningham C. T., Bardeen J. M., 1972, ApJ, 173, L137

Cunningham C. T., Bardeen J. M., 1973, ApJ, 183, 237

Davis S. W., Stone J. M., Jiang Y.-F., 2012, ApJS, 199, 9

Densmore J. D., Urbatsch T. J., Evans T. M., Buksas M. W., 2007, J. Comput. Phys., 222, 485

Dexter J., Agol E., 2009, ApJ, 696, 1616

Dibi S., Drappeau S., Fragile P. C., Markoff S., Dexter J., 2012, MNRAS, 426, 1928

Dolence J. C., Gammie C. F., Mościbrodzka M., Leung P. K., 2009, ApJS, 184,387

Dubroca B., Feugeas J. L., 1999, Comptes Rendus de l'Academie des Sciences Series I Mathematics, 329, 915

Ehlers J., 1971, in Sachs R. K., ed., General Relativity and Cosmology. Academic Press, New York, p. 1

Frolov V. P., Novikov I. D., 1998, Black Hole Physics, Basic Concepts and New Developments. Kluwer Academic Publishers, Dordrecht

González M., Audit E., Huynh P., 2007, A\&A, 464, 429

Gourgoulhon E., Jaramillo J. L., 2006, Phys. Rep., 423, 159

Hanni R. S., 1977, Phys. Rev. D, 16, 933

Hasegawa K., Umemura M., 2010, MNRAS, 407, 2632

Hayes J. C., Norman M. L., 2003, ApJS, 147, 197

Hollywood J. M., Melia F., Close L. M., McCarthy D. W., Jr, Dekeyser T. A., 1995, ApJ, 448, L21

Houck J. C., Chevalier R. A., 1991, ApJ, 376, 234

Hubeny I., Burrows A., 2007, ApJ, 659, 1458

Iliev I. T., Ciardi B., Alvarez M. A. et al., 2006, MNRAS, 371, 1057

Iliev I. T., Whalen D., Mellema G. et al., 2009, MNRAS, 400, 1283

Israel W., 1972, in O'Raifeartaigh L., ed., The relativistic Boltzmann equation - General Relativity. Clarendon Press, Oxford, p. 201

Jiang Y.-F., Stone J. M., Davis S. W., 2012, ApJS, 199, 14

Jiang Y.-F., Stone J. M., Davis S. W., 2013, ApJ, 778, 65

Jiang Y.-F., Stone J. M., Davis S. W., 2014, ApJS, 213, 7

Jiang Y.-F., Davis S. W., Stone J. M., 2016, ApJ, 827, 10

Kapoor R. C., 1981, Bull. Astron. Soc. India, 9, 232

Karas V., Bao G., 1992, A\&A, 257, 531

King A. R., Lasota J. P., Kundt W., 1975, Phys. Rev. D, 12, 3037

Kunasz P., Auer L. H., 1988, J. Quant. Spectrosc. Radiat. Transfer, 39, 67

Levermore C. D., 1984, J. Quant. Spectrosc. Radiat. Transfer, 31, 149

Levermore C. D., Pomraning G. C., 1981, ApJ, 248, 321

Li Z., Kong L., Bambi C., 2014, ApJ, 787, 152

Liebendörfer M., Rampp M., Janka H.-T., Mezzacappa A., 2005, ApJ, 620, 840

Lindquist R. W., 1966, Ann. Phys., 37, 487

McKinney J. C., Tchekhovskoy A., Sạdowski A., Narayan R., 2014, MNRAS, 411, 3177

Mellema G., Raga A. C., Canto J., Lundqvist P., Balick B., Steffen W., Noriega-Crespo A., 1998, A\&A, 331, 335

Meyer L., Eckart A., Schödel R., Duschl W. J., Mužić K., Dovčiak M., Karas V., 2006, A\&A, 460, 15

Mezzacappa A., Bruenn S. W., 1993, ApJ, 405, 669

Mezzacappa A., Liebendörfer M., Messer O. E., Hix W. R., Thielemann F.-K., Bruenn S. W., 2001, Phys. Rev. Lett., 86, 1935

Mihalas D., Mihalas B. W., 1984, Foundations of Radiation Hydrodynamics. Oxford Univ. Press, New York

Morel J. E., Wareing T. A., Lowrie R. B., Parsons D. K., 2003, Nucl. Sci. Eng., 144, 1

Mościbrodzka M., Gammie C. F., Dolence J. C., Shiokawa H., 2011, ApJ, 735,9

Mościbrodzka M., Falcke H., Shiokawa H., Gammie C. F., 2014, A\&A, 570, 7

Mościbrodzka M., Falcke H., Shiokawa H., 2016, A\&A, 586, 38

Nagakura H., Sumiyoshi K., Yamada S., 2014, ApJS, 214, 16

Nakamoto T., Umemura M., Susa H., 2001, MNRAS, 321, 593

Narayan R., Zhu Y., Psaltis D., Sądowski A., 2016, MNRAS, 457, 608

Ohsuga K., Mineshige S., 2007, ApJ, 670, 1283

Ohsuga K., Mineshige S., 2011, ApJ, 736, 2

Ohsuga K., Mineshige S., 2014, Space Sci. Rev., 183, 353

Ohsuga K., Takahashi H. R., 2016, ApJ, 818, 162
Okamoto T., Yoshikawa K., Umemura M., 2012, MNRAS, 419, 2855

Ott C. D., Burrows A., Dessart L., Livne E., 2008, ApJ, 685, 1069

Peres B., Penner A. J., Novak J., Bonazzola S., 2014, Class. Quantum Gravity, 31, 045012

Pineault S., 1977, MNRAS, 179, 691

Pineault S., Roeder R. C., 1977, ApJ, 213, 548

Pomraning G. C., 1973, The Equations of Radiation Hydrodynamics. Pergamon Press, New York

Rauch K. P., Blandford R. D., 1994, ApJ, 421, 46

Razoumov A. O., Cardall C. Y., 2005, MNRAS, 362, 1413

Ryan B. R., Dolence J. C., Gammie C. F., 2015, ApJ, 807, 31

Sachs R. K., Ehlers J., 1968, in Chrétien M., Deser S., Goldstein J., eds, Astrophysics and General Relativity Vol. 2, Brandeis University, Summer Institute in Theoretical Physics. Gordon and Breach, New York, p. 331

Schnittman J. D., Bertschinger E., 2004, ApJ, 606, 1098

Shakura N. I., Sunyaev R. A., 1973, A\&A, 24, 337

Shibata M., Sekiguchi Y., 2012, Prog. Theor. Phys., 127, 535

Skinner M. A., Ostriker E. C., 2013, ApJS, 206, 21

Sokasian A., Abel T., Hernquist L. E., 2001, New Astron., 6, 359

Steinacker J., Baes M., Gordon K. D., 2013, ARA\&A, 51, 63

Stone J. M., Mihalas D., Norman M. L., 1992, ApJS, 80, 819

Stuchlík Z., Bao G., 1992, Gen. Relativ. Gravit., 24, 945

Sumiyoshi K., Yamada S., 2012, ApJS, 199, 17

Sumiyoshi K., Yamada S., Suzuki H., Shen H., Chiba S., Toki H., 2005, ApJ, 629, 922

Sumiyoshi K., Yamada S., Suzuki H., 2007, ApJ, 667, 382

Sumiyoshi K., Takiwaki T., Matsufuru H., Yamada S., 2015, ApJS, 216, 5

Sunyaev R. A., Titarchuk L. G., 1980, A\&A, 86, 121

Susa H., 2006, PASJ, 58, 445

Sądowski A., Narayan R., 2015, MNRAS, 454, 2372

Sạdowski A., Narayan R., 2016, MNRAS, 456, 3929

Sạdowski A., Narayan R., Tchekhovskoy A., Zhu Y., 2013, MNRAS, 429, 3533

Takahashi R., 2004, ApJ, 611, 996

Takahashi R., 2007, MNRAS, 382, 567

Takahashi R., 2008, MNRAS, 383, 1155

Takahashi H., Ohsuga K., 2013, ApJ, 772, 127

Takahashi H., Ohsuga K., 2015, PASJ, 67, 60

Takahashi M., Ishizuka T., Yokosawa M., 1990, Prog. Theor. Phys., 84, 875

Turner N. J., Stone J. M., 2001, ApJS, 135, 95

Vincent F. H., Paumard T., Gourgoulhon E., Perrin G., 2011, Class. Quantum Gravity, 28, 225011

Wald M. R., 1984, General Relativity. The University of Chicago Press Ltd., London

Whitney B. A., 2011, Bull. Astron. Soc. India, 39, 101

Yoshikawa K., Yoshida N., Umemura M., 2013, ApJ, 762, 116

Younsi Z., Wu K., Fuerst S. V., 2012, A\&A, 545, 13

Yusef-Zadeh F., Morris M., White R. L., 1984, ApJ, 278, 186

Zakharov A. F., 1994, MNRAS, 269, 283

Zhang F., Zimmerman A., Nichols D. A., Chen Y., Lovelace G., Matthews K., Owen R., Thorne K. S., 2012, Phys. Rev. D, 86, 084049

Zhu Y., Narayan R., Sadowski A., Psaltis D., 2015, MNRAS, 451, 1661

\section{APPENDIX A: GR RAY-TRACING CODE: MASTER}

In order to calculate the null geodesics, a Hamiltonian formalism for photons is used (e.g. Schnittman \& Bertschinger 2004; Dolence et al. 2009). In this formalism, some conserved quantities along the geodesics explicitly appear in calculations. A Hamiltonian function $\mathcal{H}$ can be defined as a function of eight phase space variable $\left(x^{\mu}, k_{\mu}\right)$ and a variable $\lambda$ along the path length (affine parameter). Here, $x^{\mu}$ is a four vector of a position and $k_{\mu}$ is a four momentum of photon with the normalization of $k^{\mu} k_{\mu}=0$. For a general space-time metric $g_{\mu \nu}$ (with its inverse $\left.g^{\mu \nu}\right)$, a Hamiltonian function $\mathcal{H}\left(x^{\mu}, k_{\mu} ; \lambda\right)$ can be 


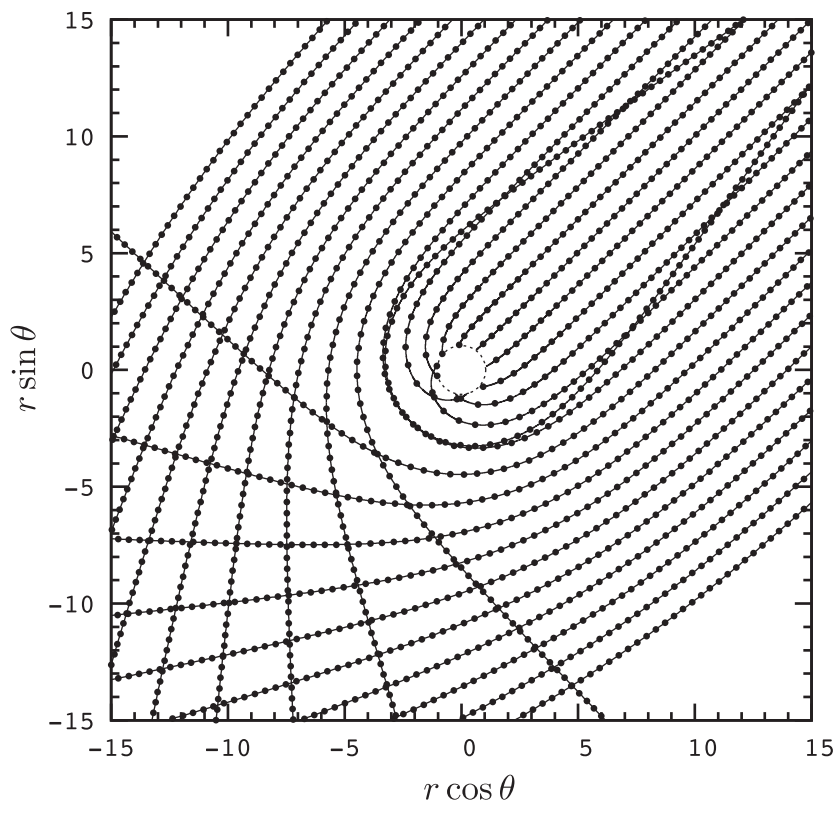

Figure A1. Null geodesics propagating backwards from an observer located at $45^{\circ}$ from the rotation axis of the black hole with $a / M=0.5$. The results of MASTER are shown by solid lines and the analytical solutions given by Rauch $\&$ Blandford (1994) are shown by dots. The location of the event horizon is shown by a dashed circle.

defined as $\mathcal{H}\left(x^{\mu}, k_{\mu} ; \lambda\right)=(1 / 2) g^{\mu v} k_{\mu} k_{v}=0$. From the Hamilton's equations given by

$\frac{\mathrm{d} x^{\mu}}{\mathrm{d} \lambda}=\frac{\partial \mathcal{H}}{\partial k_{\mu}}, \quad \frac{\mathrm{d} k_{\mu}}{\mathrm{d} \lambda}=-\frac{\partial \mathcal{H}}{\partial x^{\mu}}$,

we obtain the geodesic equations as

$\frac{\mathrm{d} x^{\mu}}{\mathrm{d} \lambda}=k^{\mu}, \quad \frac{\mathrm{d} k_{\mu}}{\mathrm{d} \lambda}=-\frac{1}{2} \frac{\partial g^{\alpha \beta}}{\partial x^{\mu}} k_{\alpha} k_{\beta}$,

where $k^{\mu}=g^{\mu v} k_{\nu}$. In this study, we numerically solve equation (A2) in the Kerr space-time. In this appendix, we verify our GR ray-tracing code, MASTER, by comparing the numerical results obtained by MASTER with the analytic solutions or the numerical results reported in the past studies. Since most of the past studies performed the ray-tracing calculations in the BL coordinates, we assume the BL coordinates in the present section.

As the first test, we compare the null geodesics calculated by MASTER with the analytical solutions given by Rauch \& Blandford (1994) and the code in Takahashi (2004). In Fig. A1, the numerically obtained geodesics by MASTER (dotted lines) and the analytic solutions (solid lines) are shown. Null geodesics are calculated for the impact parameters with $\alpha=0$ and $\beta$ in the range of $-15 \leq$ $\beta \leq 15$ with a constant interval $\Delta \beta=1$. As a result, we can confirm that MASTER successfully reproduces the analytic solutions.

The appearance of black hole shadow seen by a distant observer can be a useful test for a ray-tracing code. We first calculate the image of the affine parameter evaluated at the termination of the geodesics. This test was originally done by Dexter \& Agol (2009) and the analytic solutions of the contours of black hole shadows are given in Bardeen (1973). The results by MASTER are shown in Fig. A2 and we can confirm that MASTER successfully reproduces the past studies.

As a more realistic issue, we test MASTER for a geometricallythin accretion disc around a Kerr black hole. Such calculations have been performed by many authors in the past studies. We com- (a)

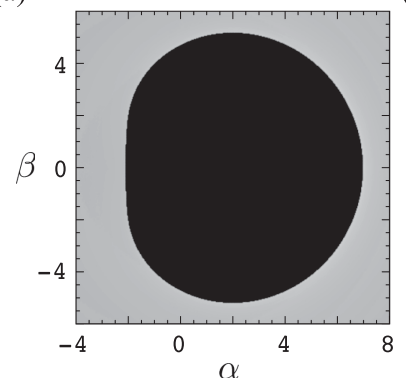

(b)

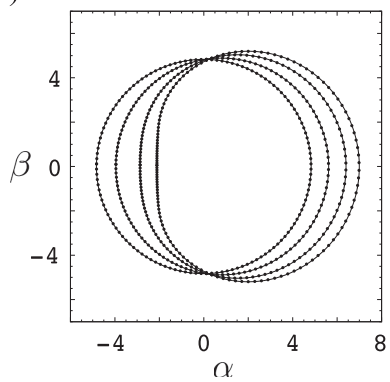

Figure A2. (a) Image in affine parameter for a Kerr black hole with $a / M=0.998$ viewed by an observer located at the equatorial plane, reproducing fig. 2 of Dexter \& Agol (2009). (b) Contours of the shadow of Kerr black hole with $a / M=1$ calculated by an analytic formula in Bardeen (1973) (solid lines) and by MASTER (dots) for the viewing angles $i=0^{\circ}, 20^{\circ}$, $45^{\circ}$ and $90^{\circ}$.
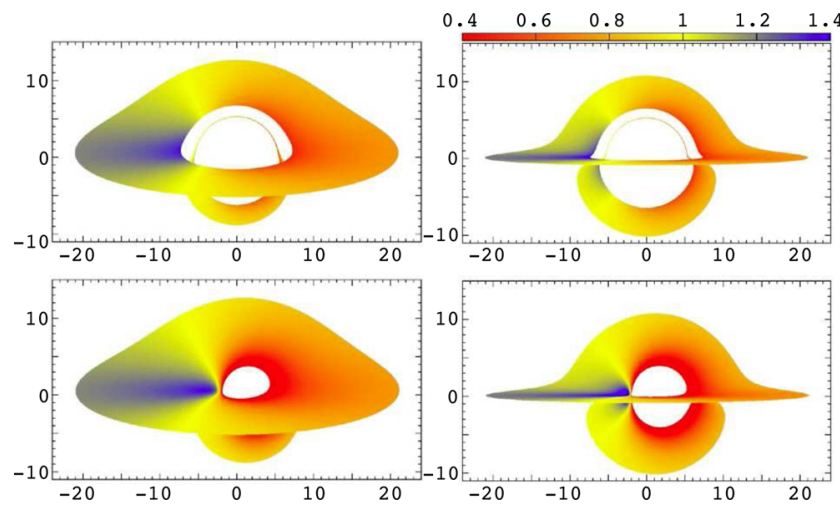

Figure A3. Maps of redshift functions, i.e. $g$-factor $\left(g=v_{o} / v_{e}\right)$, of a thin disc around a black hole with $a / M=0$ (top) and 1 (bottom) seen by a distant observer. The viewing angle is $i=75^{\circ}$ and 87.5 (left to right). The inner and outer boundaries of the disc are $r_{\text {in }} / M=r_{\text {ISCO }}$ and $r_{\text {out }} / M=20$, respectively. These are similar to fig. 6 of Beckwith \& Done (2005).

pare our results to those by Bromley, Chen \& Miller (1997) and Beckwith \& Done (2005) for the images and to those by Schnittman \& Bertschinger (2004) and Dexter \& Agol (2009) for the energy spectrum. In Fig. A3, we show a map produced by direct orbits and relativistic orbits for the selected parameters of viewing angles. We can confirm that MASTER can reproduce the results in Beckwith \& Done (2005). We also calculate the energy spectra of thin disc. We solve the same problems done by Schnittman \& Bertschinger (2004) and Dexter \& Agol (2009). We calculate the energy spectra with two different resolutions: one is the calculations with $1000^{2}$ rays and the other $8000^{2}$ rays. The results are shown in Fig. A4 and we obtain the results similar to those in Schnittman \& Bertschinger (2004) and Dexter \& Agol (2009). As shown in the top panel of Fig. A4, in the results produced by $1000^{2}$ rays, we can see small jaggies in the spectra. These unfavourable features disappear, if we use $8000^{2}$ rays shown in the bottom panel of Fig. A4.

The problem of a hotspot orbiting a black hole is of great significance and applicable for a wide variety of phenomena around the black hole. This problem has been investigated by many authors in the past studies (e.g. Cunningham \& Bardeen 1972, 1973; Pineault 1977; Pineault \& Roeder 1977; Kapoor 1981; Bao 1992; Karas \& Bao 1992; Stuchlik \& Bao 1992; Bao et al. 1994; Zakharov 1994; Hollywood et al. 1995; Broderick \& Loeb 2005, 2006; Meyer et al. 2006; Li et al. 2014). A hotspot model has 

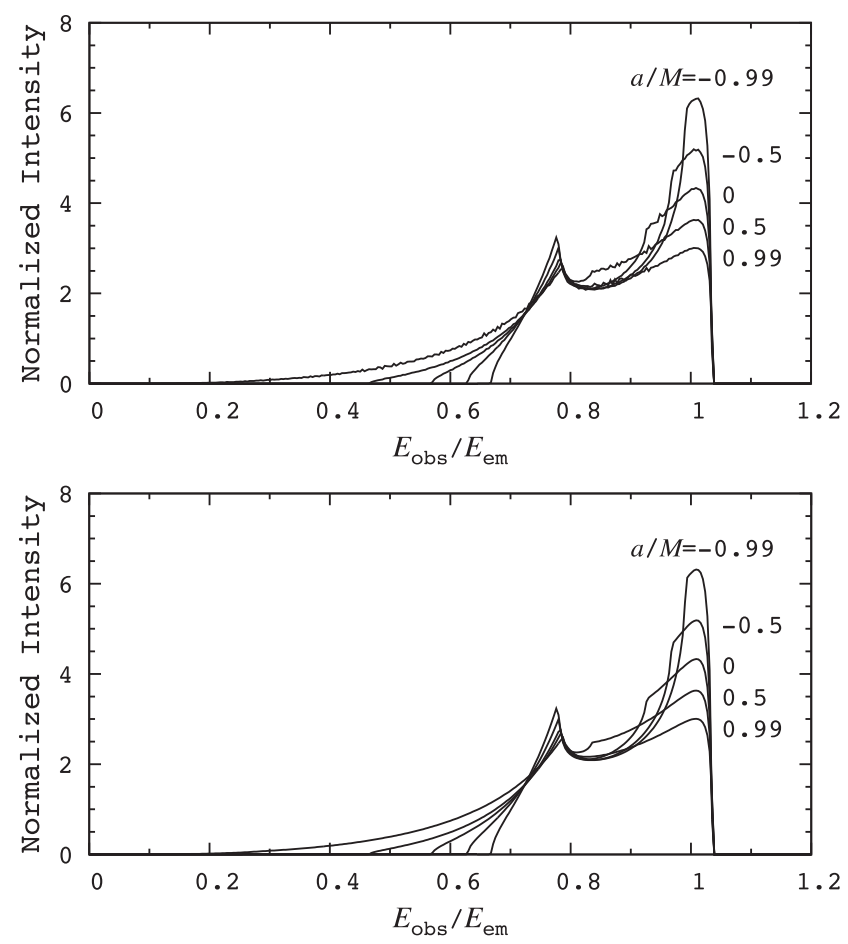

Figure A4. Normalized energy spectra of a thin disc observed with a viewing angle $i=30^{\circ}$ for the Kerr parameter $a / M=-0.99,-0.5,0,0.5$, and 0.99 . The inner and outer boundary of the disc are $r_{\text {in }} / M=r_{\text {ISCO }}$ and $r_{\text {out }} / M=15$, respectively. The emissivity is assumed to be proportional to $r^{-2}$. Calculations are performed with $1000^{2}$ rays (top) and $8000^{2}$ rays (bottom). These match fig. 4 of Schnittman \& Bertschinger (2004) and fig. 7 of Dexter \& Agol (2009).

been fairly successful in explaining the observed time-variability of accreting black holes. We apply MASTER for this problem in the Schwarzschild space-time. A simplified model for this test is given in Schnittman \& Bertschinger (2004) and Dexter \& Agol (2009). The same problem as these two papers is solved by MASTER. In the left-hand panel in Fig. A5, we show the spectrogram of an orbiting hotspot at the ISCO viewed from $i=60^{\circ}$. The results are similar to those in Schnittman \& Bertschinger (2004) and Dexter \& Agol (2009). In the right-hand panel in Fig. A5, we show the results calculated by MASTER. In these tests, we can obtain the results similar to those in Schnittman \& Bertschinger (2004) and Dexter \& Agol (2009). (a)

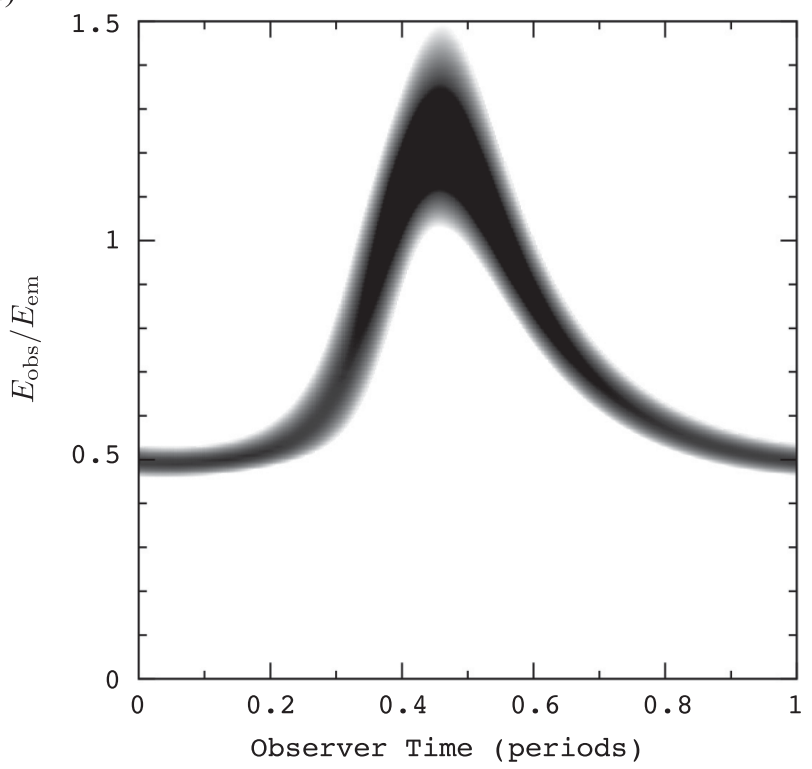

(b)

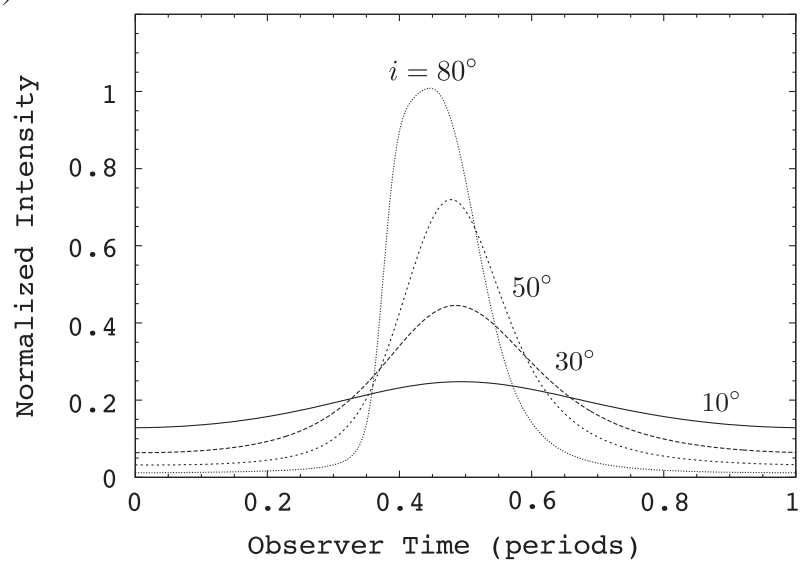

Figure A5. (a) Spectrogram of an orbiting hotspot at the ISCO viewed from the location with a viewing angle $i=60^{\circ}$. This can be compared to fig. 4 of Schnittman \& Bertschinger (2004) and fig. 6 of Dexter \& Agol (2009). (b) Frequency-integrated light curves of an orbiting hotspot at the ISCO viewed from the location with a viewing angle $i=10^{\circ}, 30^{\circ}, 50^{\circ}$ and $80^{\circ}$. This should be compared to fig. 3 of Schnittman \& Bertschinger (2004) and fig. 5 of Dexter \& Agol (2009).

This paper has been typeset from a $\mathrm{T}_{\mathrm{E}} \mathrm{X} / \mathrm{L} \mathrm{T} \mathrm{E} \mathrm{X}$ file prepared by the author. 